Draft VERSion June 13, 2021

Preprint typeset using $\mathrm{L}^{\mathrm{A}} \mathrm{T} \mathrm{E}$ X style emulateapj v. 01/23/15

\title{
REMOVING BIASES IN RESOLVED STELLAR MASS-MAPS OF GALAXY DISKS THROUGH SUCCESSIVE BAYESIAN MARGINALIZATION
}

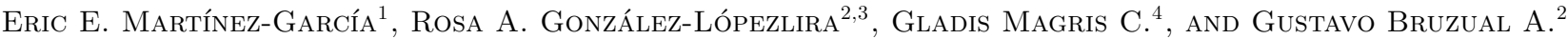 \\ 1 Cerrada del Rey 40-A, Chimalcoyoc Tlalpan, Ciudad de México, C.P. 14630; martinezgarciaeric@gmail.com \\ 2 Instituto de Radioastronomía y Astrofísica, UNAM, Campus Morelia, Michoacán, México, C.P. 58089 \\ 3 Argelander Institut für Astronomie, Universität Bonn, Auf dem Hügel 71, D-53121 Bonn, Germany and \\ 4 Centro de Investigaciones de Astronomía, Apartado Postal 264, Mérida 5101-A, Venezuela \\ Draft version June 13, 2021
}

\begin{abstract}
Stellar masses of galaxies are frequently obtained by fitting stellar population synthesis models to galaxy photometry or spectra. The state of the art method resolves spatial structures within a galaxy to assess the total stellar mass content. In comparison to unresolved studies, resolved methods yield, on average, higher fractions of stellar mass for galaxies. In this work we improve the current method in order to mitigate a bias related to the resolved spatial distribution derived for the mass. The bias consists in an apparent filamentary mass distribution, and a spatial coincidence between mass structures and dust lanes near spiral arms. The improved method is based on iterative Bayesian marginalization, through a new algorithm we have named Bayesian Successive Priors (BSP). We have applied BSP to M 51, and to a pilot sample of 90 spiral galaxies from the Ohio State University Bright Spiral Galaxy Survey. By comparing quantitatively both methods, we find that the average fraction of stellar mass missed by unresolved studies is only half than previously thought. In contrast with the previous method, the output BSP mass-maps bear a better resemblance to near infrared images. Subject headings: galaxies: fundamental parameters — galaxies: stellar content — galaxies: photometry — galaxies: spiral — methods: statistical
\end{abstract}

\section{INTRODUCTION}

How galaxies form and assemble their mass is a primordial question in modern astrophysics. Galaxy masses are crucial for their evolution, and for the evolution of cosmic structures at all scales. The determination of the stellar mass content of galaxies can help constrain, e.g., the dark matter fraction, the specific star formation rate ( $\Psi_{\mathrm{S}}$, the star formation rate, $\Psi$, per unit stellar mass), the stellar mass function, and the universe's stellar mass density and star formation history ( $\mathrm{SFH}$ ).

There are different methods to estimate the mass of a galaxy, e.g., dynamical or through gravitational lensing (see Courteau et al. 2014, for a review). Regarding the stellar mass component, the use of stellar population synthesis (SPS) models to estimate mass through the stellar mass-to-light ratio, $\Upsilon_{*} \sqrt{1}$, has been frequently advocated (e.g. Bell \& de Jong 2001; Bell et al. 2003). Notwithstanding their common degeneracies, SPS models can in general yield reliable mass estimates. One novel technique is the resolved stellar mass-map method ( $\mathrm{Zi}-$ betti, Charlot, \& Rix 2009, ZCR hereafter), that delivers a map of the stellar mass surface density by photometric means. Galaxy masses determined by treating the galaxies as point sources are often underestimated (and sometimes overestimated, see Roediger \& Courteau 2015), thus the need to resolve structures (ZCR; Sorba \& Sawicki 2015). Even more, if the stellar mass of each galaxy in a cluster is estimated separately, the total stellar mass fraction is lower than when a constant $\Upsilon_{*}$ is assumed (Leauthaud et al. 2012).

1 Throughout this work $\Upsilon_{*}$ refers to the stellar (including remnants) mass-to-light ratio in units of $M_{\odot} / L_{\odot}$, i.e., we do not include dark matter, nor gas mass in $\Upsilon_{*}$.
The resolved stellar mass method is truly powerful, since it can solve not only for the mass, but for other physical parameters of the SPS models, based solely on photometry. Resolved maps of stellar mass are also important for studies aimed at understanding the dynamics of bars and/or spirals (since gravity is the main driver), and their secular evolution (e.g., Foyle et al. 2010, Martínez-García \& González-Lópezlira 2013: Egusa et al. 2016). Additionally, they can be used to determine the baryonic contribution to rotation curves (e.g., Repetto et al. 2013, 2015, McGaugh et al. 2016). The method can also be extended to higher-redshift studies (e.g., LanyonFoster et al. 2007; Wuyts et al. 2012).

Despite their potential, the resulting mass-maps may be biased, in the sense that the stellar mass shows a filamentary structure and is concentrated in dust lanes. In this paper we aim to understand the origin of this shortcoming and improve the method to derive resolved stellar mass-maps. We must also mention that in this research we use SPS models that assume a constant metallicity along the SFH. Gallazzi \& Bell (2009) studied the effects of using a variable metallicity SPS library and found no significant biases when estimating $\Upsilon_{*}$. Nevertheless, Into \& Portinari (2013) indicate that the color-mass-to-light ratio relations (CMLR, see e.g. McGaugh \& Schombert 2014 ) resulting from an evolving metallicity along a coherent SFH within an individual galaxy are probably different from the CMLR established for the general galaxy population. Furthermore, biases in mass determinations from CMLR can be even more significant at high redshifts than for local studies (see e.g., Mitchell et al.2013). In this work we do not use CMLR to recover $\Upsilon_{*}$; instead, we use a statistically robust Bayesian technique to infer the predicted $\Upsilon_{*}$ via the comparison of observed colors 
with a comprehensive library of SPS models.

The paper is organized as follows. In section 2 we describe the resolved stellar mass-map method in its present form and explain/investigate the source of the bias. We introduce a new method (based on the former) in section 3. In section 4 we apply the new method to the spiral galaxy M 51 (NGC 5194); comparisons with other methods are also briefly described. In section 5 we apply the new method to a pilot sample of spiral galaxies, and discuss and analyze the results. The uncertainties in the stellar mass estimates are discussed in section 6. Finally, we give our conclusions in section 7 .

\section{RESOLVED MAPS OF STELLAR MASS}

The ZCR method uses a Monte Carlo library of SPS models obtained from the 2007 version of Bruzual \& Charlot (2003; CB07) models with the Chabrier (2003) stellar initial mass function (IMF). The library was built by adopting prior probability distributions for parameters such as the SFH, the dust attenuation (treated as in the two-component model of Charlot \& Fall 2000), and a non-evolving metallicity. By randomly drawing the parameters from the prior distributions (cf. da Cunha et al. 2008), the resulting library consists of $\approx 5 \times 10^{4}$ templates (or models).

The ZCR fiducial method is based on surface brightness photometry at the $g$ and $i$ Sloan Digital Sky Survey (SDSS) optical bands, and one near-infrared (NIR) filter such as $J, H$, or $K$. The method was extended to include the Spitzer Space Telescope Infrared Array Camera (IRAC) $3.6 \mu \mathrm{m}$-band by Repetto et al. (2015). Other optical color combinations are possible, with the disadvantage of having more degeneracy in $\Upsilon_{*}$, and thus more uncertain results (see e.g., Repetto et al. 2015, Bell \& de Jong 2001, their Figures 1 and 2, respectively). The templates from the SPS library are binned in colors $(g-i)$ and $(i-H)$, using a bin width of 0.05 magnitude (see Figure 1). The median mass-to-light ratio at the $H$ band, $\Upsilon_{*}^{H}$, is estimated for each bin. A look-up table can thus be constructed to compare with observed photometry on a pixel-by-pixel basis. The $\Upsilon_{*}^{H}$ is the effective mass-to-light ratio, i.e., refers to the light that reaches the observer, as opposed to the light that is emitted. The effective $\Upsilon_{*}^{H}$ may be affected by extinction (ZCR).

Earlier studies concerning pixel-by-pixel spatially resolved properties of galaxies can be found in Bothun (1986), Abraham et al. (1999), Conti et al. (2003), Eskridge et al. (2003), Kassin et al. (2003), Lanyon-Foster et al. (2007), and Welikala et al. (2008).

\subsection{Application to M 51. A filamentary mass structure?}

Now we present results obtained by applying the ZCR method to the spiral galaxy M 51. We use $g$ and $i$ band imaging from the twelfth data release (DR12) of the SDSS (Alam et al. 2015), as well as the $K_{s}$-band mosaic from Gonzalez \& Graham (1996). The NIR images were obtained at Kitt Peak National Observatory (KPNO), with the IR Imager (IRIM) camera on the 1.3 meter telescope; the IRIM had a $256^{2}$ NICMOS3 array with a $2^{\prime \prime}$ pixel $^{-1}$ plate scale. The observations were performed during March 1994, in non-photometric conditions, and the exposures were resampled with subpixel accuracy before combining. The final $K_{s}$-band mosaic has $0.5 \times 0.5 \operatorname{arcsec}^{2}$ pixels, and a total exposure time of 22 minutes; it was photometrically calibrated ${ }^{2}$ with the Two Micron All Sky Survey (2MASS, Skrutskie et al. 2006). The SDSS frames were re-sampled to the resolution of the NIR data, and registered with the $K_{s}$-band image. The registration was done with the IRAF ${ }^{3}$ (Tody 1993) tasks GEOMAP and GREGISTER. No point spread function (PSF) match was done to the images, since the data have similar PSFs and the process can corrupt the noise properties (Zibetti, Charlot, \& Rix 2009). In Figures 2a (top left panel), and 2b (top right panel), we show the $K_{s}$-band and $g$-band final images, respectively. The foreground stars and background galaxies were removed and their pixels replaced with values from the background-subtracted "sky". With the purpose of isolating the disk from the lower signal-to-noise $(\mathrm{S} / \mathrm{N})$ background, the final mosaics were treated with the Adaptsmooth code of Zibetti (2009), as follows. A first run of Adaptsmooth was performed on the the $K_{s^{-}}$ band data (which have a lower $\mathrm{S} / \mathrm{N}$ ratio than the SDSS images), with the requirement of a minimum $\mathrm{S} / \mathrm{N}$ ratio per pixel of 20, a maximum smoothing radius of 10 , and the assumption of background-dominated noise. In order to homogenize the lower limit of the $\mathrm{S} / \mathrm{N}$ ratio per pixel, the output smoothing $K_{s}$-band mask was then used as an input, in subsequent runs of Adaptsmooth, for the SDSS $g$ and $i$ bands.

The SPS library was obtained from the Multiwavelength Analysis of Galaxy Physical Properties package (MAGPHYS-CB07 library, hereafter) by da Cunha et al. (2008) ${ }^{4}$ The absolute magnitudes of the Sun were taken from Blanton \& Roweis (2007). We assume a distance to M 51 of $9.9 \pm 0.7 \mathrm{Mpc}$ ('Tikhonov et al. 2009), and correct the models for Galactic extinction (Schlafly \& Finkbeiner 2011).

The resulting mass-map is presented in Figure 2. (bottom left panel). For comparison purposes we show in Figure 3 the $i$-band image. The color range covered by the observed photometry of M 51 is shown, as a 2-D histogram, in Figure 4. In the left panel we show the observed colors of the pixels after applying the Adaptsmooth procedure as described earlier. The right panel shows the observed colors of the same pixels without using the Adaptsmooth procedure. From the comparison of these plots we appreciate the advantage of increasing the $\mathrm{S} / \mathrm{N}$ ratio in the outskirts of the disk, otherwise the uncertainties in the fits would be quite large. In these figures we also demarcate the color range covered by $99 \%$ and $68 \%$ of the total templates in the MAGPHYS-CB07 library, with a blue and a red contour, respectively. Most of the observed colors fall within the span of the SPS library. The plots are illustrative and do not reflect the observational uncertainties of the data.

One striking thing to notice about the mass-map (Fig-

2 Throughout this work NIR magnitudes are Vega, SDSS magnitudes are in the $\mathrm{AB}$ magnitude system.

3 IRAF is distributed by the National Optical Astronomy Observatories, which are operated by the Association of Universities for Research in Astronomy, Inc., under cooperative agreement with the National Science Foundation.

4 http://www.iap.fr/magphys/magphys/MAGPHYS.html 

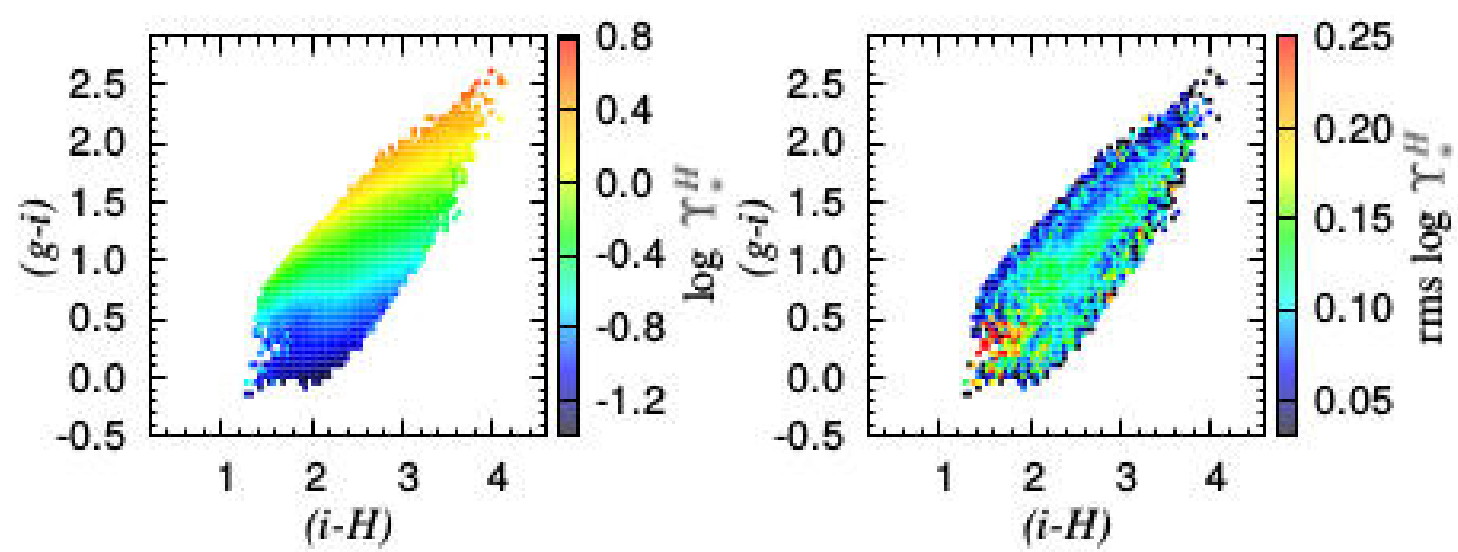

FIG. 1. - Left: decimal logarithm of the effective, i.e., as seen by the observer (cf. ZCR), mass-to-light ratio at the $H$ band, $\Upsilon_{*}^{H}$, derived from the $(g-i)$ vs. $(i-H)$ color-color diagram. The data are taken from the MAGPHYS-CB07 Monte Carlo SPS library, and grouped in bins $0.05 \times 0.05 \mathrm{mag}^{2}$. SDSS $g$ and $i$ magnitudes are in the AB magnitude system, $H$ magnitudes are Vega. Right: root mean square (rms) errors of $\log \Upsilon_{*}^{H}$ in the left panel. The global median rms error is 0.1 dex.
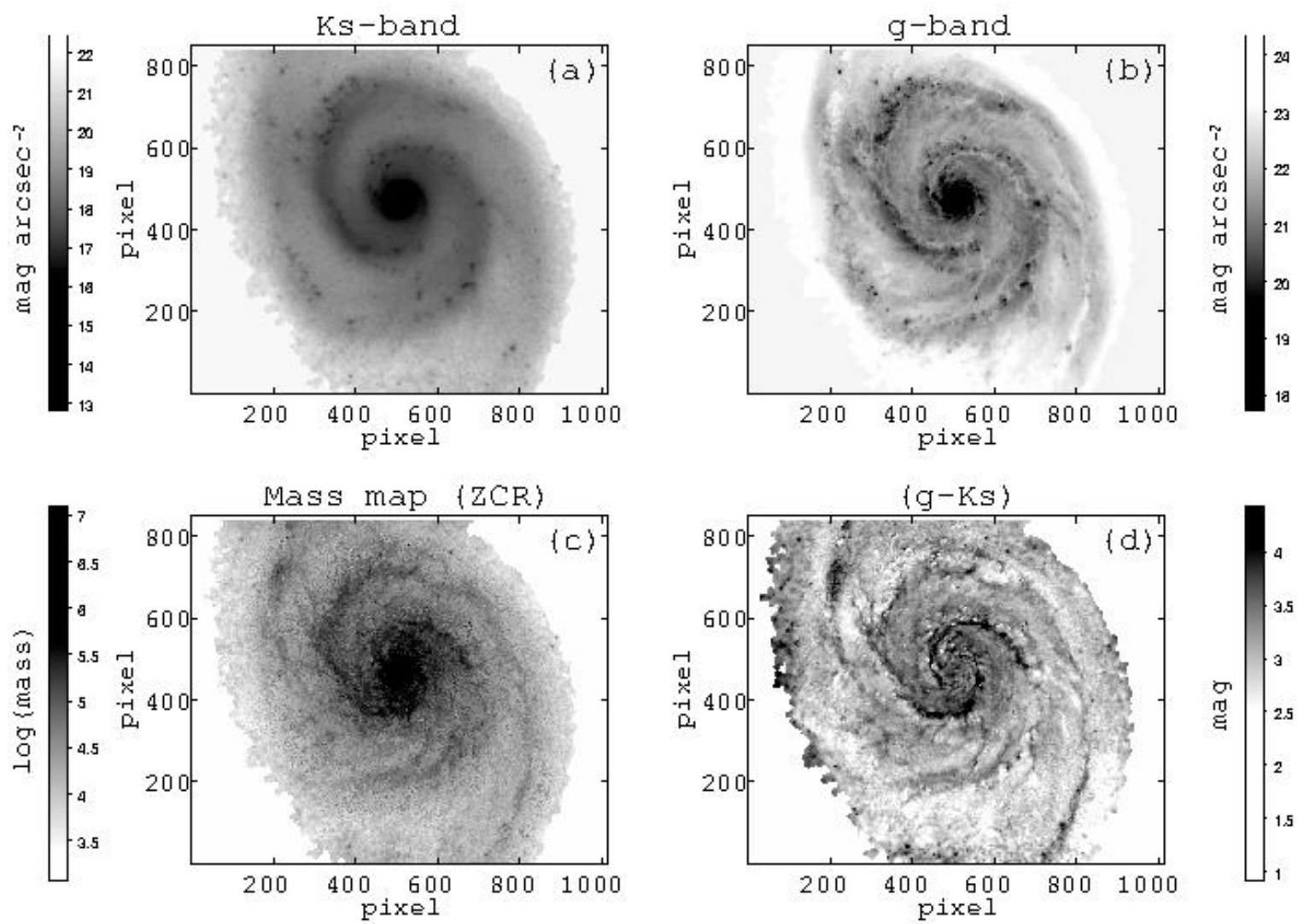

Fig. 2.- Top left: $K_{s}$-band mosaic of M 51; grayscale in Vega mag. Top right: $g$-band mosaic of M 51 ; grayscale in AB mag. Bottom left: M 51 stellar mass-map derived with the ZCR method, based on $(g-i)$ and $\left(i-K_{s}\right)$ colors, and $K_{s}$ mass-to-light ratio, $\Upsilon_{*}^{K_{s}}$; mass in $M_{\odot}$. Bottom right: $\left(g-K_{s}\right)$ color map of M 51. Notice the similarities of the features in this extinction map and in the stellar mass-map in the bottom left panel (c). Higher extinction is indicated by darker features. North is up, East is to the left. 


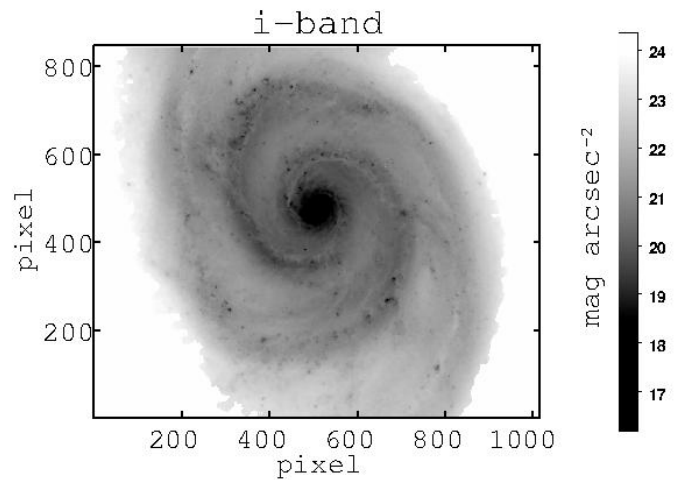

FIG. 3.- SDSS $i$-band mosaic of M 51. Grayscale in AB mag. North is up, East is to the left.

ure 25) is that it does not present a smooth spiral arm structure. There is a well defined two-arm spiral pattern, but many filamentary structures are also observed. In addition, a visual comparison of the mass structure with the optical $g$-band indicates that, presumably, most of the structure is coincident with the dust lanes, as inferred from optical extinction. This can be seen more easily in Figure $2 \mathrm{~d}$ (bottom right panel), where we show the $\left(g-K_{s}\right)$ image. To test the similarities between the mass-map and the $\left(g-K_{s}\right)$ image quantitatively, we use cross-correlation techniques. The Pearson correlation coefficient is defined as

$$
r=\frac{\sum_{j} \sum_{i}\left(f_{i j}-\bar{f}\right)\left(g_{i j}-\bar{g}\right)}{\sqrt{\sum_{j} \sum_{i}\left(f_{i j}-\bar{f}\right)^{2}} \sqrt{\sum_{j} \sum_{i}\left(g_{i j}-\bar{g}\right)^{2}}},
$$

where $f_{i j}$ is the intensity of the $i^{\text {th }}, j^{\text {th }}$ pixel in the first image, $g_{i j}$ is the intensity of the $i^{\text {th }}, j^{\text {th }}$ pixel in the second image, $\bar{f}$ is the mean intensity of the first image, and $\bar{g}$ is the mean intensity of the second image. The cross-correlation function, $(f \star g)(\theta)$, is then obtained by rotating the first image with respect to the second one, while fixing the center of rotation at the center of the object (the nuclei of M 51 in this case). We obtain $r(\theta)$ from equation 1 by varying $\theta$ from $-180^{\circ}$ to $180^{\circ}$ in increments of $1^{\circ}$; we assume that the angle $\theta$ increases counterclockwise. All the M 51 data were deprojected assuming an inclination angle of $20^{\circ}$, and a position angle of $172^{\circ}$ (Leroy et al. 2008). The result of the crosscorrelation between the output mass-map of the ZCR method and the intensity ratio in the $\left(g-K_{s}\right)$ image is shown in Figure 5. By "intensity ratio", we mean the ratio between the intensity in the $g$-band image and the intensity in the $K_{s}$-band image. We use this ratio instead of the $\left(g-K_{s}\right)$ color because the latter scales logarithmically and cannot be compared with the mass distribution, that scales linearly. Note that we actually take the intensity ratio in the minus $\left(g-K_{s}\right)$ image; this is done with the purpose of getting positive values of $r$ (when using equation 1). Error bars were estimated with bootstrap methods (Bhavsar 1990; Lepage \& Billard 1992). We replace each pixel separately with a random value, drawn from a Gaussian probability distribution, and for each $\theta$ recalculate equation 1 . We repeat this process a total of
30 times and calculate $\sigma_{c c}$, the standard deviation of the resulting distribution.

There is clearly a peak in the cross-correlation function near $\theta=0^{\circ}$, indicating a similarity between the structures. For comparison, we also show the cross-correlation between the intensity in the $K_{s}$-band image and the intensity ratio in the $\left(g-K_{s}\right)$ image. The absolute maximum in this case occurs around $\theta=-15.5^{\circ} \pm 0.8$, and marks the angular offset between the spiral arms in the $K_{s}$-band and the dust lanes in the $\left(g-K_{s}\right)$ image. This means that if we rotate the spiral arms in the $K_{s}$-band by $15^{\circ}$, clockwise, they will match the spatial location of the dust lanes.

As is well known, disk galaxies, when studied at different wavelengths, often show significant differences (e.g., Block \& Wainscoat 1991, Block et al. 1994). Even if at NIR wavelengths young stars and clusters can contribute $20 \%-30 \%$ to the total radiation in spiral arm regions (e.g., Rix \& Rieke 1993, Gonzalez \& Graham 1996 Rhoads 1998; James \& Seigar 1999, Patsis et al. 2001 Grosbøl et al. 2006, Grosbøl \& Dottori 2008), most of the light in the disk comes from evolved giant stars, and most of the mass is concentrated in low mass main sequence stars. Hence, any structures present in resolved stellar mass-maps should resemble the NIR surface brightness morphology to a significant degree. This is not the case of the stellar mass-map shown in Figure 2a (bottom left panel), where we see filamentary structure not present in the $K_{s}$ light distribution, Figure 2 (top left panel).

We perform three other different and independent tests, and compare the resulting stellar mass-maps as described below.

1. We do not use the NIR band, and rely only on the optical SDSS colors, e.g., $(u-i)$ and $(g-i)$, and on the mass-to-light ratio estimated in the $i$-band, $\Upsilon_{*}^{i}$.

2. We remove the binning of the models and use the full $5 \times 10^{4}$ templates of the MAGPHYS-CB07 library in the computations.

3. We use a new Monte Carlo SPS (optical-NIR) library taken from the Synthetic Spectral Atlas of Galaxies (SSAG; Magris et al. 2015). SSAG ${ }^{5}$ assumes random SFHs according to the Chen et al. (2012) prescription, that includes a burst and a truncation event. Dust is treated as in Charlot \& Fall (2000), and metallicity is distributed between $0.02 Z_{\odot}$ and $2.5 Z_{\odot}$, with $95 \%$ galaxy templates having $Z>0.2 Z \odot$. The adopted IMF is Chabrier. The library contains $6.7 \times 10^{4}$ templates (SSAG-BC03 library henceforth). The range in these models of the effective mass-to-light ratio in the $K_{s}$-band, $\Upsilon_{*}^{K_{s}}$, as determined by a $(g-i)$ vs. $\left(i-K_{s}\right)$ color-color diagram, is shown in Figure 6. left panel. For comparison purposes we show the same diagram for the $\mathrm{BC} 03$ version of the MAGPHYS library (MAGPHYS-BC03) in the right panel. The MAGPHYS library extends to redder colors due to the different probability distribution functions used to model the optical depth in the $V$-band, $\tau_{V}$ (see Figure 7).

\footnotetext{
5 http://www.astro.ljmu.ac.uk/ asticabr/SSAG.html
} 

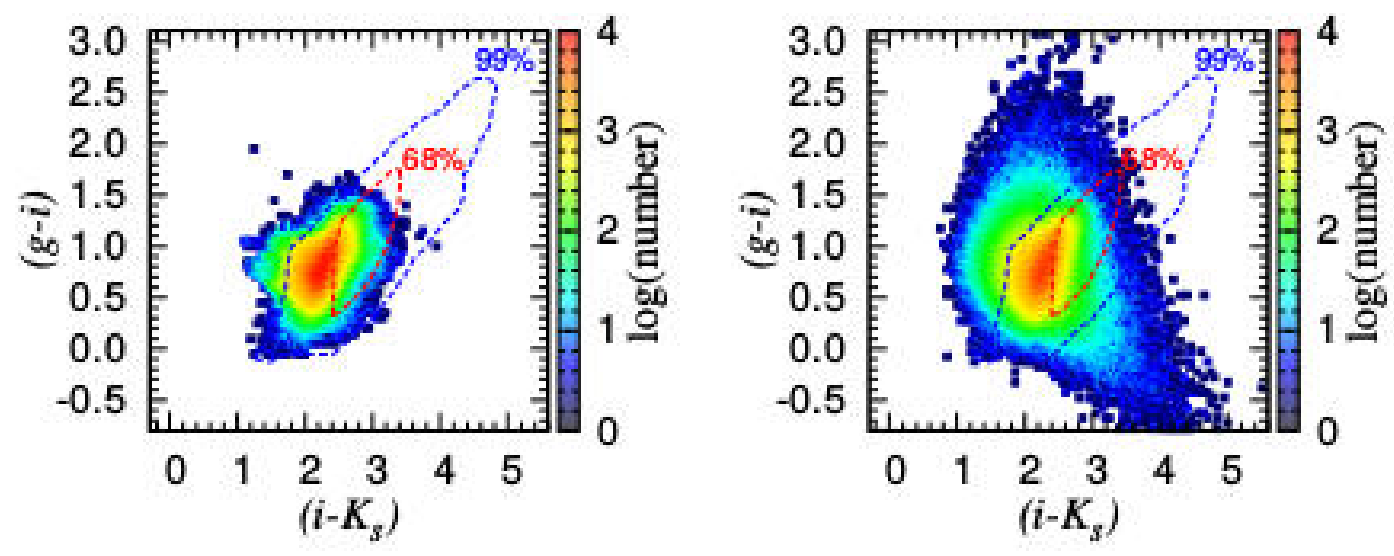

FIG. 4. - 2-D histograms of the observed $(g-i)$ and $\left(i-K_{s}\right)$ colors of M 51's pixels. The areas inside the blue and red dashed lines contain $99 \%$ and $68 \%$, respectively, of the templates in the MAGPHYS-CB07 SPS library corrected for Galactic extinction. Left: after applying the Adaptsmooth procedure as described in the text. The maximum of $\log \left(\right.$ number) occurs near $\left(i-K_{s}\right) \sim 2.29$ and $(g-i) \sim 0.85$. Right: without applying the Adaptsmooth procedure.

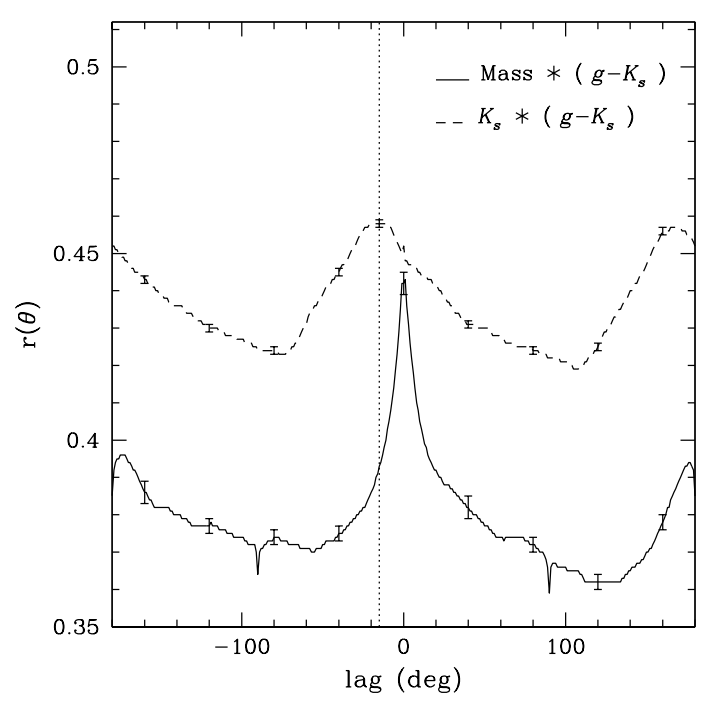

FIG. 5.- Cross correlation functions $r(\theta)$ (see text). Solid line: between the mass structure resulting from the ZCR method and the intensity ratio in the $\left(g-K_{s}\right)$ image. The absolute maximum is at $\theta=0^{\circ}$, indicating similarity. Dashed line: between the intensity in the $K_{s}$-band image and the intensity ratio in the $\left(g-K_{s}\right)$ extinction map. The maximum occurs at $\theta \sim-15^{\circ}$ (marked by the vertical dotted line), and corresponds to the angular lag between the dust lanes and the stellar arms. The total height of each error bar is $2 \sigma_{c c}$.

The filamentary structure, and the spatial coincidence between mass and dust lanes prevail in all the tests. A similar result is obtained for other spiral galaxies as well, and was already noticed although not sufficiently discussed in ZCR (their Figure 8). It is noteworthy that this is not a problem of resolution in the SPS libraries, since the mean sampling is $\sim 0.005 \%$ or less, both for colors and for $\Upsilon_{*}^{K_{s}}$; hence, the template set is densely populated.

In this work we will focus on the structural properties of the mass-maps. We will only mention here that both the local and the integrated stellar masses derived from SPS models may vary on account of different treatments of the thermally-pulsating asymptotic giant branch (TP-
AGB; see, e.g., Maraston et al. 2006 Bruzual 2007: Conroy et al. 2009), and the choice of IMF in the libraries. The mass determinations may also differ if obtained from different bands, even when using the same models (McGaugh \& Schombert 2014).

\subsection{The level of accuracy in mass-to-light ratio estimates}

Gallazzi \& Bell (2009) discuss thoroughly the $\Upsilon_{*}$ accuracy that can be achieved by comparing colors with predictions from a large library of SFHs. Typical accuracies are of the order of 0.1-0.15 dex. A similar result is deduced by other authors (e.g., Bell \& de Jong 2001; ZCR; Taylor et al. 2011). This level of accuracy is barely improved with spectroscopic data (Gallazzi \& Bell 2009).

To better understand the impact of a limited $\Upsilon_{*}$ accuracy on the resolved mass-maps of galaxies, we build a sample of mock galaxies drawn from the MAGPHYSCB07 Monte Carlo SPS library. Each of the $\approx 5 \times 10^{4}$ templates is used as an individual object in our mock catalog. In order to simulate the photometric error, we add to each of the $g, i$, and $K_{s}$-band magnitudes in our mocks a random noise component with a Gaussian distribution, having $\sigma_{\mathrm{mag}}=0.02 \mathrm{mag}(\sim 2 \%$ intensity variation $)$. We then try to fit the noisy $(g-i)$ and $\left(i-K_{s}\right)$ values of each simulated object with the noise-free $(g-i)$ and $\left(i-K_{s}\right)$ colors, via $\chi^{2}$ minimization. Afterwards we compute

$$
\Delta \log \left[\Upsilon_{*}^{K_{s}}\right]=\log \left[\Upsilon_{*}^{K_{s}}\right]_{\text {fit }}-\log \left[\Upsilon_{*}^{K_{s}}\right]_{\text {true }},
$$

i.e., the ratio between the fitted $\Upsilon_{*}$ and the true value. The results of this test are shown in Figure $8{ }^{6}$ where we get a dispersion (standard deviation) $\sigma\left(\Delta \log \left[\Upsilon_{*}^{K_{s}}\right]\right) \sim$ 0.16 dex, as expected. We carry out the same exercise for different $\sigma_{\text {mag }}$ values and obtain $\sigma\left(\Delta \log \left[\Upsilon_{*}^{K_{s}}\right]\right)$ for each one. The results are shown in Figure 9, upper panel. There is a nearly linear decrease of $\sigma\left(\Delta \log \left[\Upsilon_{*}^{K_{s}}\right]\right)$ with diminishing $\sigma_{\text {mag }}$ down to $\sigma_{\text {mag }} \sim 0.005$. For lower values of $\sigma_{\text {mag }}$, the shape of the $\Delta \log \left[\Upsilon_{*}^{K_{s}}\right]$ distribution

6 We notice that Gallazzi \& Bell (2009) obtain a similar plot in spite of neglecting dust corrections, which indicates that dust is not a decisive factor for $\Upsilon_{*}$ accuracy. 

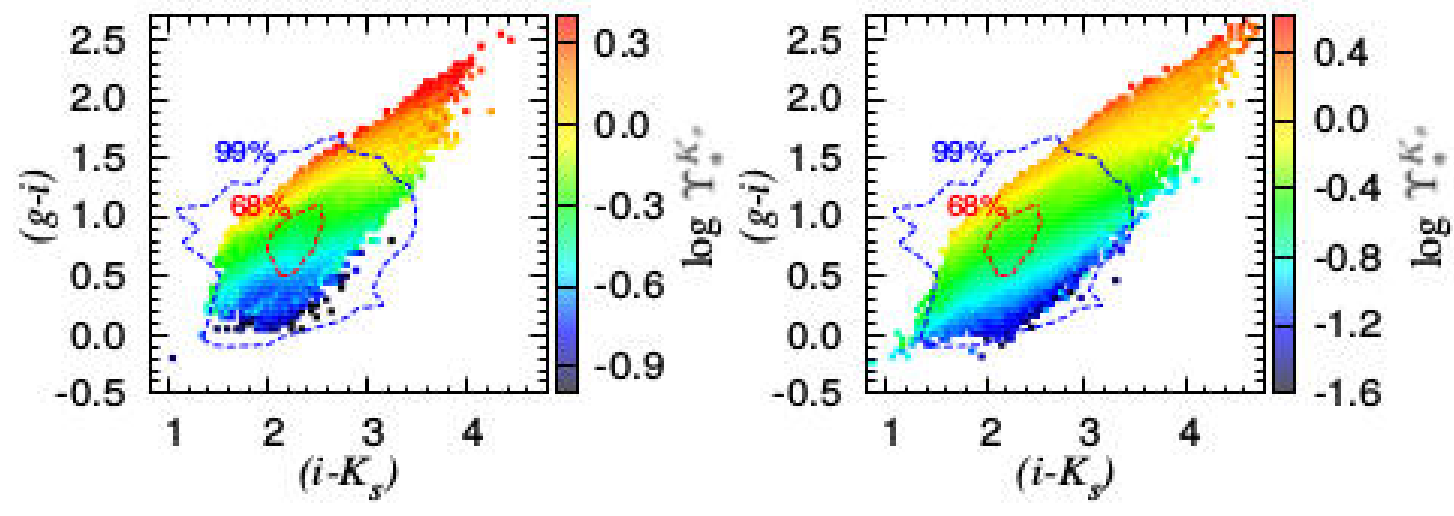

FIG. 6. - Left: decimal logarithm of the effective mass-to-light ratio at the $K_{s}$-band, $\Upsilon_{*}^{K_{s}}$, derived from the $(g-i)$ vs. $\left(i-K_{s}\right)$ color-color diagram. The data are taken from the SSAG-BC03 Monte Carlo SPS library (Magris et al. 2015), corrected for Galactic extinction towards $\mathrm{M}$ 51. SDSS $g$ and $i$ magnitudes are in the AB magnitude system, $K_{s}$ magnitudes are vega. The blue/red dashed contour delimits 99\%/68\% of the observed colors for M 51 (see Figure 4 left panel). Right: analogous to left panel, but for the MAGPHYS-BC03 Monte Carlo SPS library.

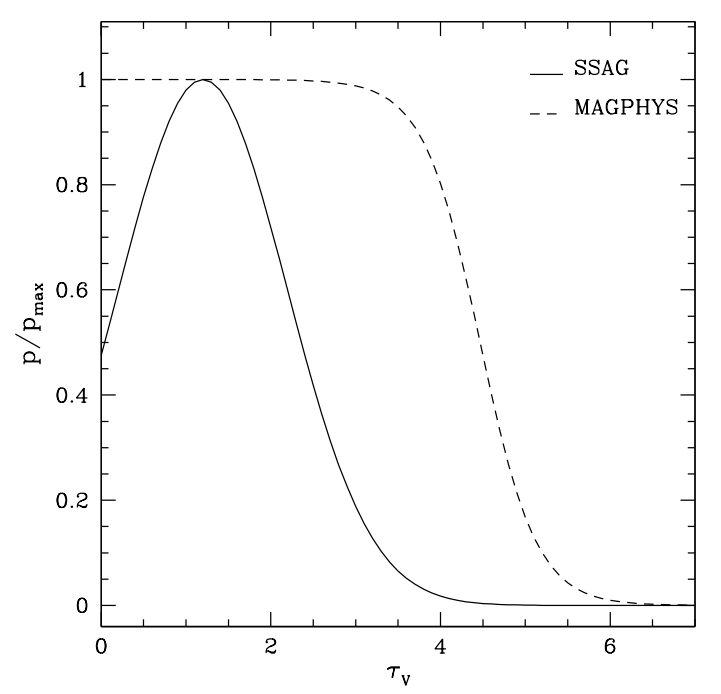

FIG. 7.- Probability distribution functions of the $V$-band optical depth of the dust seen by young stars, $\tau_{V}$, used by the SSAG-BC03 (solid line), and the MAGPHYS (dashed line) Monte Carlo SPS libraries, respectively.

abruptly begins to change, from nearly Gaussian with kurtosis $\sim 3$, going through Laplace distributions, and finally tending to a Dirac delta function with kurtosis $\rightarrow \infty$. This effect can be appreciated in the lower panel of Figure 9 , where we plot the excess kurtosis 7 of $\Delta \log \left[\Upsilon_{*}^{K_{s}}\right]$ versus $\sigma_{\text {mag }}$.

As $\sigma_{\text {mag }}$ tends to zero, the dispersion, $\sigma\left(\Delta \log \left[\Upsilon_{*}^{K_{s}}\right]\right)$, also tends to zero. A (hypothetical) value of $\sigma\left(\Delta \log \left[\Upsilon_{*}^{K_{s}}\right]\right)=0$ would indicate that our adjusted values are equal to the true values (the noise-free models). We can infer that it is not feasible to get accurate $\Upsilon_{*}$ values unless the intrinsic errors of the observations are diminished to zero, i.e., $\sigma_{\mathrm{mag}} \rightarrow 0$. Typical photometric calibration errors are of the order of $1-2 \%$ for the

\footnotetext{
7 Excess kurtosis is measured with respect to the kurtosis of any univariate normal distribution, which equals 3. Therefore, excess kurtosis equals kurtosis minus 3.
}

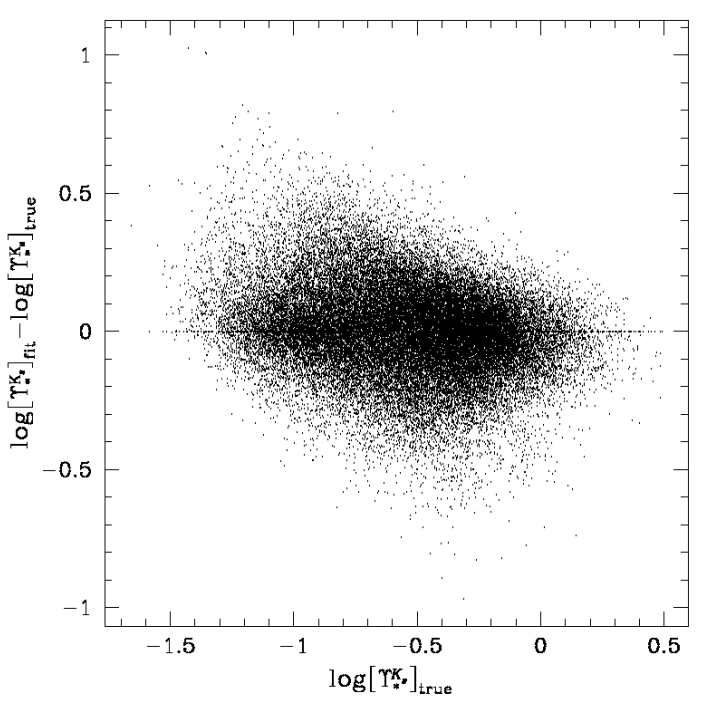

FIG. 8. - Fits to colors of $\approx 5 \times 10^{4}$ mock galaxies (see text). Noise modeled as a random Gaussian distribution with $\sigma_{\mathrm{mag}}=0.02 \mathrm{mag}$ is added to the mock objects before fitting them with the noise-free templates of the MAGPHYS-CB07 library. The difference is quantified as $\Delta \log \left[\Upsilon_{*}^{K_{s}}\right]=\log \left[\Upsilon_{*}^{K_{s}}\right]_{\text {fit }}-\log \left[\Upsilon_{*}^{K_{s}}\right]_{\text {true }}$. The standard deviation of $\Delta \log \left[\Upsilon_{*}^{K_{s}}\right]$ is $\sigma\left(\Delta \log \left[\Upsilon_{*}^{K_{s}}\right]\right)=0.16$ dex.

SDSS (Padmanabhan et al. 2008) and other photometric surveys. Additionally to this, the degeneracies between the different SPS model parameters (e.g., age-metallicityreddening) will prevail even when $\sigma_{\mathrm{mag}} \rightarrow 0$.

Taking all this into account we can conclude that the features in resolved mass-maps, acquired from a simple $\chi^{2}$ minimization, will be discrepant from the structures of NIR surface brightness maps, owing to a limited $\Upsilon_{*}$ accuracy. In this manner, the fit we can obtain for some observed colors will result in a $\Upsilon_{*}$ value near the statistical mode of similar colors in the SPS library (see also the discussion in Taylor et al. 2011), and within $0.1-0.15$ dex of the true value. Even for the same SPS library, the "recovered" $\Upsilon_{*}$ will depend on the colors used in the fit. 

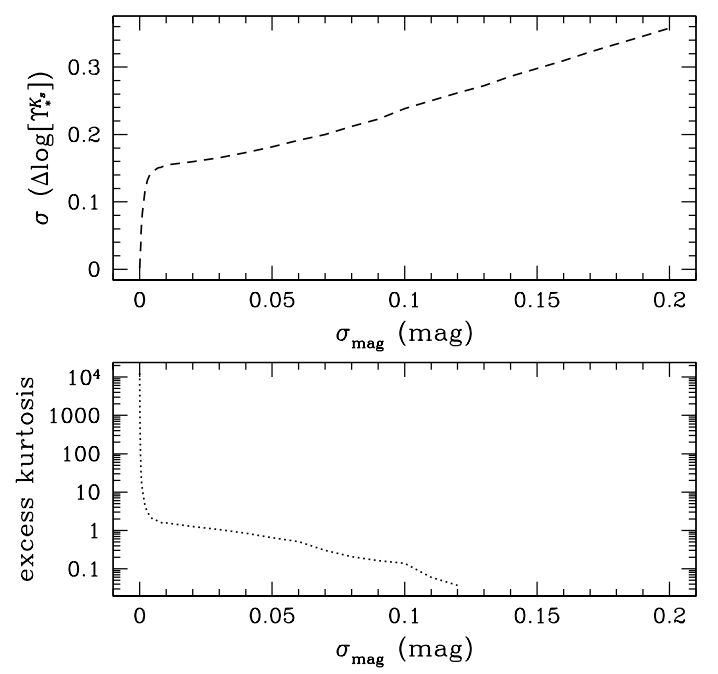

$$
P\left(\Upsilon_{*} \mid C\right)=\frac{P\left(C \mid \Upsilon_{*}\right) P\left(\Upsilon_{*}\right)}{P(C)}
$$

where $P\left(\Upsilon_{*} \mid C\right)$ is the posterior probability, i.e., the probability of having $\Upsilon_{*}$, for a certain stellar population, if colors $C$ are observed.

$P\left(C \mid \Upsilon_{*}\right)$ is the likelihood function (or the probability of observing colors $C$ given the set of parameters $\Upsilon_{*}$ ):

$$
\begin{gathered}
P\left(C \mid \Upsilon_{*}\right) \propto \frac{1}{\sqrt{2 \pi}} \exp \left(-\frac{\chi^{2}}{2}\right), \\
\chi^{2}=\sum_{n=1}^{N_{\text {colors }}}\left(\frac{C_{n}^{\text {obs }}-C_{n}^{\text {template }}}{\sigma_{\text {col }}}\right)^{2},
\end{gathered}
$$

where $C_{n}^{\text {obs }}$ is the observed $n_{\text {th }}$ color with $\sigma_{\text {col }}$ photometric error, and $C_{n}^{\text {template }}$ is the color from a certain template in our SPS library. In our case $N_{\text {colors }}=2$, for instance, $(g-i)$ and $\left(i-K_{s}\right)$, hence $n=1,2$.

$P\left(\Upsilon_{*}\right)$ represents the previous knowledge we may have

FIG. 9. - Statistical parameters of the fits to colors of mock galaxies. Top: standard deviation, $\sigma\left(\Delta \log \left[\Upsilon_{*}^{K_{s}}\right]\right)$, vs. $\sigma_{\text {mag }}$ in the range 0.0-0.2 magnitude (see also Figure 8 where $\sigma_{\text {mag }}=$ 0.02 magnitude). Bottom: excess kurtosis (or kurtosis minus 3 ) of $\Delta \log \left[\Upsilon_{*}^{K_{s}}\right]$ vs. $\sigma_{\mathrm{mag}}$.

\section{BAYESIAN INFERENCE AIMED AT AN OBJECT}

In this section we introduce the Bayesian successive priors (BSP) algorithm, aimed at an individual object, in order to solve for the mass-map avoiding the bias in the spatial structure. The idea is to use the previous information regarding the stellar surface mass density as deduced from the NIR bands. The massive older population of a galaxy is mainly traced in the NIR bands, specially the $K$-band (Rix \& Rieke 1993). Having established this, we can adopt the NIR surface brightness distribution as a Bayesian prior, in order to infer the "true" stellar surface mass density. In this work, we will use the term "prior" in reference to the prior probability distribution function. The Bayesian prior is then directed to a particular galaxy, and not to the entire galaxy population.

\subsection{Bayes' theorem}

Bayesian probability posits that the best outcome of any event is found by calculating the probabilities of the various hypotheses involved, using the rules of probability theory (e.g., Loredo 1992, 1995).

The ZCR approach uses a method similar to a Bayesian maximum-likelihood estimate by including a uniform (or flat) prior in the fits to the observed colors, regardless of the SPS library. In the present work a significant improvement is made in the calculation of the stellar mass-maps, by introducing a Bayesian method with an informative, non-uniform, prior. Applications of Bayesian inference with non-uniform priors have been used in, e.g., Benítez (2000), for cosmological redshift estimates, Rovilos et al. (2014), for AGN sources analysis, and Schonrich \& Bergemann (2014), for the determination of stellar parameters.

In our case, Bayes' theorem for the most probable stellar mass-to-light ratio $\Upsilon_{*}$ is given by

about the likely value of the $\Upsilon_{*}$ parameter, and

$$
P(C)=\sum_{j=1}^{N_{\text {templates }}} P\left(C \mid \Upsilon_{* j}\right) P\left(\Upsilon_{* j}\right)
$$

is a normalization constant, also called the Bayesian evidence (Savage \& Oliver 2007). $N_{\text {templates }}$ stands for the number of templates in our SPS library.

\subsection{The Bayesian successive priors (BSP) algorithm}

\subsubsection{The prior probability distribution function}

In order to apply the BSP algorithm, we have chosen a prior probability distribution function, $P\left(\Upsilon_{*}\right)$, of the form

$$
P\left(\Upsilon_{*}\right)=\exp \left(-\frac{1}{2}\left[\frac{\Upsilon_{*}^{\text {prior }}-\Upsilon_{*}}{\sigma_{\Upsilon_{*}}}\right]^{2}\right)
$$

where

$$
\sigma_{\Upsilon_{*}}=\left[\frac{\ln (10)}{2.5}\right] \sigma_{\mathrm{mag}} \Upsilon_{*}^{\text {prior }} .
$$

Here, $\sigma_{\mathrm{mag}}$ is the photometric error for a certain passband, which is related to $\sigma_{\mathrm{col}}$ in equation 5 through $\sqrt{2} \sigma_{\mathrm{mag}} \approx \sigma_{\mathrm{col}}$.

Each template in the SPS library corresponds to a single $\Upsilon_{*}$. By using equation 7 and Bayes' theorem (equation 3), we can effectively marginalize the templates from our SPS library, as we will demonstrate in the following sections.

\subsubsection{Description of the BSP algorithm}

The BSP algorithm consists of three iterations that are described below. The algorithm is intended to work with a SPS library and surface photometry in several/various bands. In the following we assume that these are the optical $g$ and $i$ bands, and the NIR $K_{s}$ filter. For the 
library, we use SSAG-BC03 (although the algorithm is designed to work independently of the choice of SPS library). The mass-to-light ratio is taken in the $K_{s}$-band, $\Upsilon_{*}^{K_{s}}$. Other waveband combinations will be discussed later. The algorithm is applied on a pixel-by-pixel basis, although in each iteration all pixels are addressed before moving to the next iteration.

1. In the first iteration we use a uniform prior, i.e., $P\left(\Upsilon_{*}\right)=$ constant, and apply equation 3 . Then we calculate the absolute maximum (which should be near the median) of the posterior probability distribution function $P\left(\Upsilon_{*}^{K_{s}} \mid C\right)$, and the 16th and 84th percentiles, to account for the corresponding error map. We estimate the percentiles by progressively integrating the area under the posterior probability curve until we accumulate an area of 0.16 and 0.84 (being the total area equal to 1 ), for the 16 th and 84th percentiles, respectively ${ }^{8}$

Up to this point the method provides a maximum likelihood estimate and is similar to the ZCR algorithm, with the only difference that the templates are not binned in our case. We call the unbinned version of the $\mathrm{ZCR}$ algorithm $\mathrm{ZCR}^{\prime}$ from now on. We then use the results of this step for two purposes. Firstly, we identify all the pixels for which the difference (absolute value) between their observed color and the fitted template in the SPS library is smaller than $3 \sigma_{\mathrm{col}}$, i.e.,

$$
\left|\Delta C_{n}\right|=\left|C_{n}^{\text {obs }}-C_{n}^{\text {template }}\right|<3 \sigma_{\mathrm{col}},
$$

for $n=1,2$. The pixels that do not fulfill the $3 \sigma_{\text {col }}$ condition are isolated and flagged ${ }^{9}$ This step guarantees that we keep only pixels that can be described by our SPS library. Next, we take the resulting $\Upsilon_{*}^{K_{s}}$ values for all the kept pixels and calculate the statistical median 10

2. In the second iteration this median value of $\Upsilon_{*}^{K_{s}}$, from iteration number 1 , is used as a constant parameter in equation 7 , i.e.,

$$
\Upsilon_{*}^{\text {prior }}=\text { constant }
$$

for all pixels in the disk ${ }^{11}$ The prior, $P\left(\Upsilon_{*}\right)$, is not uniform in this case, and adopts the functional form of equation 7. Now we compute the maximum in $P\left(\Upsilon_{*}^{K_{s}} \mid C\right)$, and the respective 16 th and 84 th percentiles. Similarly to iteration number 1 , we identify all the pixels where the difference between the observed colors and the fitted library templates is smaller than $\alpha \sigma_{\mathrm{P}}$, i.e.,

8 These values are equivalent to $-1 \sigma$ and $1 \sigma$, respectively, in a normal distribution.

9 These include elements recording emission from AGN activity.

10 The number separating the lower and higher value halves of $\Upsilon_{*}^{K_{s}}$.

11 A refinement of the method could be achieved by separating the bulge from the disk of the galaxy, and treating them as objects with different median $\Upsilon_{*}^{K_{s}}$ (Portinari et al. 2004).

$$
\left|\Delta C_{n}\right|<\alpha \sigma_{\mathrm{P}}
$$

for $n=1,2$. The value of $\sigma_{\mathrm{P}}$ is determined from the resulting $\Delta C_{n}$ (no absolute value) pixel distribution by calculating its 16 th and 84 th percentiles, $\mathrm{P}_{16}$ and $\mathrm{P}_{84}$, respectively, and then using

$$
\sigma_{\mathrm{P}}=\left(\mathrm{P}_{84}-\mathrm{P}_{16}\right) / 2
$$

for each color. After some tests (see Appendix A, we have found that $\alpha=1.0$ is an adequate value that allows us to isolate the pixels that deviate significantly from $\Delta C_{n} \sim 0$. In a hypothetical case, having $\Delta C_{n}=0$ would indicate that our observed colors match perfectly the fitted library templates. The $\left|\Delta C_{n}\right|<\alpha \sigma_{\mathrm{P}}$ pixels will be the "backbone" of our mass-map, and represent the locations in the disk where the $K_{s}$-band is a reliable tracer of the stellar mass surface density, considering the $\Upsilon_{*}^{\text {prior }}=$ constant condition. The $\left|\Delta C_{n}\right|>\alpha \sigma_{\mathrm{P}}$ pixels belong mainly to luminous red stars in the asymptotic giant branch, red supergiants, low surface brightness regions in the outskirts of the disk, and high extinction regions where $\Upsilon_{*}^{K_{s}}$ does not have the constant (median) value we assumed earlier. We then need to provide a new $\Upsilon_{*}^{K_{s}}$ value for these $\left|\Delta C_{n}\right|>\alpha \sigma_{\mathrm{P}}$ pixels. For this purpose we use the information from the "backbone" pixels. We interpolate the stellar mass surface density to fill the places where we need a new $\Upsilon_{*}^{K_{s}}$ value. The interpolation is done in the $0^{\circ}, 45^{\circ}, 90^{\circ}$, and $135^{\circ}$ directions, and then an average is taken. After the interpolation, we visually inspect the resulting maps to determine whether a minor smoothing is needed. The smoothing is only applied to the $\left|\Delta C_{n}\right|>\alpha \sigma_{\mathrm{P}}$ pixels, and is performed by replacing each pixel value with the average of the neighboring pixels. There are other interpolation techniques that could be used (see, e.g., Gumus \& Sen 2013), but for the present work we will apply the above mentioned procedure to all objects. Having established this, the new $\Upsilon_{*}^{K_{s}}$ values are estimated as the ratio of the interpolated mass-map and the observed $K_{s}$ photometry.

3. The third and last iteration is intended to deal only with the $\left|\Delta C_{n}\right|>\alpha \sigma_{\mathrm{P}}$ pixels, identified in iteration number 2. For each pixel, we use the $\Upsilon_{*}^{K_{s}}$ value also estimated in iteration number 2 to represent $\Upsilon_{*}^{\text {prior }}$ in equation 7 , and calculate the absolute maximum of the posterior probability distribution in equation 3. Before this, we may also update the uncertainty in $\Upsilon_{*}$, in equation 7 , such uncertainty now reads

$$
\sigma_{\Upsilon_{*}}=\sqrt{\left(\left[\frac{\ln (10)}{2.5}\right] \sigma_{\operatorname{mag}} \Upsilon_{*}^{\text {prior }}\right)^{2}+\beta^{2}},
$$

where $\beta$ accounts for the propagation of uncertainties arising from the previous iteration (e.g., the 


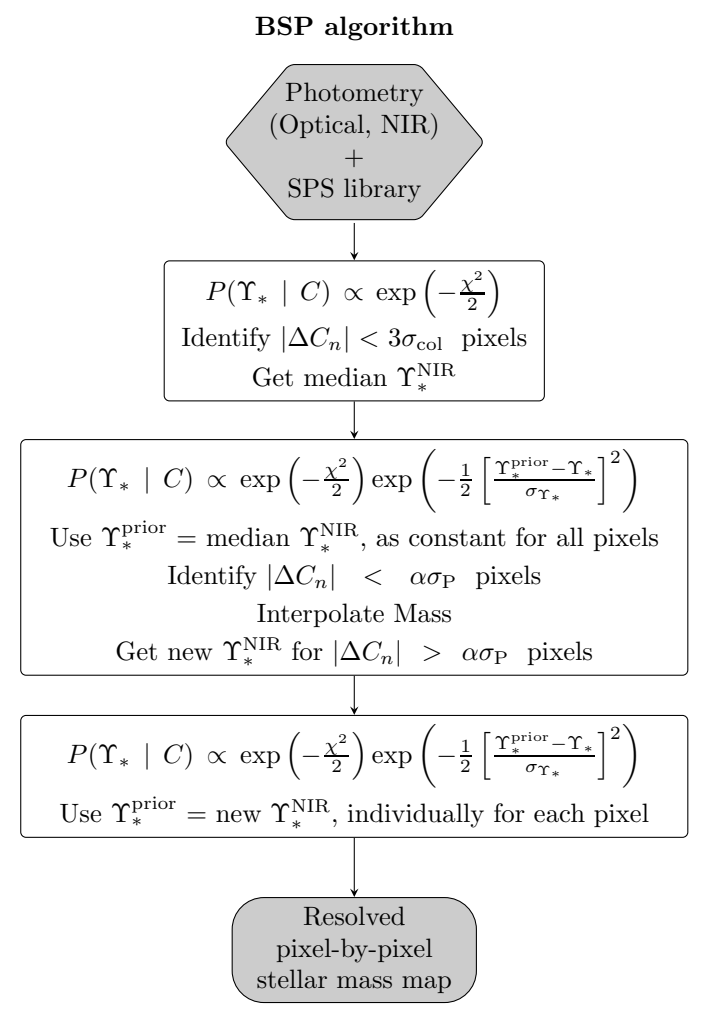

Fig. 10.- Bayesian successive priors (BSP) flowchart. Photometry in some optical bands (e.g., SDSS $g$ and $i$ ) and one NIR band (e.g., $\left.K_{s}\right)$, as well as a SPS library, are required. $P\left(\Upsilon_{*} \mid C\right)$ denotes the posterior mass-to-light ratio probability distribution; $\Delta C_{n}$ is the difference between the observed $n_{\text {th }}$ color of a pixel, and the color of the fitted template in the SPS library; $\sigma_{\mathrm{col}}$ is the photometric error in the $n_{\text {th }}$ color; $\sigma_{\mathrm{P}}$ is determined from the $\Delta C_{n}$ pixel distribution in iteration number 2 (see equation 12. Each one of the three rectangular white boxes stands for an iteration of the BSP algorithm (see text).

mass surface density interpolation from neighboring pixels). Using bootstrap methods we have estimated that $\beta \approx 0.6 \%$.

From the resulting $\Upsilon_{*}^{K_{s}}$ map we then obtain the stellar mass surface density to complete our massmap.

As an optional last step, the flagged pixels from iteration number 1 that belong to the inner disk can be interpolated in mass with the information about the surrounding pixels provided by all three iterations. For the external disk pixels, the interpolation is more uncertain.

We find that adding more iterations does not lead to any further improvement in the mass-maps. The flowchart of the BSP algorithm is shown in Figure 10

For the BSP algorithm to work properly, the requirement of NIR data with high $\mathrm{S} / \mathrm{N}$ ratio is essential; otherwise, any noisy and patchy features will be transferred to the mass-map. A minimum $\mathrm{S} / \mathrm{N}$ ratio of $\sim 10-20$ in the outskirts of the disk is necessary. This level can be achieved with techniques as the one used by the Adaptsmooth code, or alternatively with Voronoi twodimensional binning (Cappellari \& Copin 2003).

In this investigation we have adopted only two colors, $(g-i)$ and $\left(i-K_{s}\right)$, and thus $N_{\text {colors }}=2$. The benefits of using the $g$ and $i$ SDSS data together with one
NIR band are an excellent spatial resolution per element (pixel), and extensive spatial coverage (of the entire object). Nevertheless, the BSP algorithm can also be applied by using $N_{\text {colors }}>2$, with the only requirement of the inclusion of one NIR band as described earlier. In a separate publication we will explore the use of the algorithm to fit optical IFU observations, for instance, the Calar Alto Legacy Integral Field Area survey (CALIFA, Sánchez et al. 2012), and the Mapping Nearby Galaxies at Apache Point Observatory survey (MaNGA, Bundy et al. 2015).

\section{APPLICATION OF BSP TO M 51}

We apply the BSP algorithm to M 51 employing the same data described in section 2.1. We calculate $\sigma_{\text {mag }}$ on a pixel-by-pixel basis assuming that

$$
\sigma_{\text {mag }} \approx \sqrt{\sigma_{\text {flux }}^{2}+\sigma_{\text {calib }}^{2}},
$$

where $\sigma_{\text {flux }}$ is the random error in the flux per pixel, which we assume to be dominated by the uncertainty in the background (see also, Mentuch Cooper et al. 2012), and $\sigma_{\text {calib }}$ is the calibration uncertainty, or zero point error, for which we assume $\sigma_{\text {calib }} \sim 0.01$ mag for the SDSS images, and $\sigma_{\text {calib }} \sim 0.03 \mathrm{mag}$ for the $K_{s}$ image (Jarrett et al. 2003). We compute $\sigma_{\text {flux }}$ in mag by using $\sigma_{\text {flux }}=1.085736 * \frac{\sigma_{\text {back }}}{\text { flux }}$, where $\sigma_{\text {back }}$ is the standard deviation in the background (in a sky-subtracted image). We compute $\sigma_{\text {back }}$ by sampling the background statistics in different boxes near the edges of the images. To account for the use of the Adaptsmooth procedure we divide $\sigma_{\text {back }}$ by $\sqrt{n_{\text {pix }}}$, where $n_{\text {pix }}$ is the number of pixels used to increase the $\mathrm{S} / \mathrm{N}$ of the corresponding pixel by Adaptsmooth.

Without taking into account correlation between bands, we compute $\sigma_{\text {col }}$ by summing in quadrature the $\sigma_{\text {mag }}$ values of each band involved in the color determination.

In Figures 11 and 12, we show the results of adopting the SSAG-BC03 and MAGPHYS-CB07 libraries, respectively. In both figures, the top left panels (a) show the mask obtained after iteration number 1 . White regions represent the pixels where the observed colors are within $3 \sigma$ of at least one SPS-library template (see Figures 4 or 6). In the respective top right panels (b), we show the masks obtained after iteration number 2 . For these masks, the gray regions represent the pixels where the color difference (absolute value) between the models and the observations, $\left|\Delta C_{n}\right|$, is greater than $\alpha \sigma_{\mathrm{P}}$, with $\alpha=1$ (see section 3.2, and Appendix A), assuming a constant $\Upsilon_{*}^{K_{s}}$. These regions will be interpolated in mass with the information of neighboring pixels. We can also appreciate that the SSAG-BC03 library does a better job at modeling the outskirts of the disk than the MAGPHYSCB07 library. To investigate the cause of this behavior we obtain a mass map by using MAGPHYS-BC03. We obtain very similar masks to those from the SSAG-BC03 library (Figure 11, top panels). With this in mind, most of the differences between BC03 and CB07 mass-maps in our results are mainly due to the distinct treatments of the TP-AGB stage. To a lesser extent, we also notice an improvement when SSAG-BC03 is used, instead 
of MAGPHYS-BC03. We attribute this to the fact that SSAG covers a wider range of possible star formation histories.

In the bottom left panels (c) of Figures 11 and 12 , we show the resulting stellar mass surface density map after iteration number 3 . The filamentary structure is no longer present, and the maps show greater resemblance to the features in NIR bands, as expected. Finally, the bottom right panels (d) of both figures show the "residuals"; these are the result of subtracting the final output (iteration 3) mass-map using BSP, from a mass-map that assumes a constant $\Upsilon_{*}^{K_{s}}$ (the median $\Upsilon_{*}$ after iteration number 1 ). The dark/white regions represent positive/negative mass differences, i.e., where $\Upsilon_{*}$ has been overestimated/underestimated. For example, the $\Upsilon_{*}$ may be overestimated when young luminous red stars are mixed with older populations, and underestimated due to extinction in the NIR bands. This is different from the "outshining bias" (Maraston et al. 2010. Sorba \& Sawicki 2015), where the light from young stars eclipses the old population and the amount of stellar mass is underestimated. In our case we overestimate the mass (by using a constant $\Upsilon_{*}^{K_{s}}$ ) because we are assuming, mistakenly but for convenience, that all the light comes from old stars.

\subsection{Isolating the old massive disk}

We will now discuss in more detail the positive mass differences in the residuals. In Figure 13 we plot a 2-D histogram of the colors of the pixels for which the mass difference is $>2 \times 10^{4} M_{\odot}$. This cut in the mass was chosen in order to isolate most of the positive residuals near the spiral arms. We have excluded the pixels from the bulge region. We note that most points gather in a group with a maximum near $\left(i-K_{s}\right) \sim 2.4$ and $(g-$ $i) \sim 0.4$. Their $(g-i)$ color is relatively blue when compared with all the colors observed (delimited by the blue dashed contour). We also note a cluster of points with redder colors, near $\left(i-K_{s}\right) \sim 2.5$ and $(g-i) \sim 1.3$. These pixels mainly correspond to point sources outside the spiral arms.

In Figure 14 we show the marginalized probability distributions (see Appendix B for the $r$-band lightweighted age and for $\Upsilon_{*}^{K_{s}}$, obtained for M 51 using the MAGPHYS-CB07 library. The dashed-dotted green line corresponds to the, previously described, "positive mass differences" in the residuals, while the blue solid line refers to the whole disk, both results after BSP. Interestingly, the excess mass regions are younger (age $\sim 1$ Gyr) and have a lower $\Upsilon_{*}^{K_{s}}$ (by 30\%) than most of the pixels in the disk. Together with the bluer $(g-i)$ color, the above characteristics indicate that these regions contain relatively young stars, that mix with the old stellar population in star forming regions. These were effectively isolated by BSP!

The red dashed line in Figure 14 shows the probability distributions for the whole disk after applying the $\mathrm{ZCR}^{\prime}$ approach. The light-weighted age yields a larger fraction of younger pixels with ZCR'. As expected from our previous assumptions, the values of $\Upsilon_{*}^{K_{s}}$ are more narrowly confined with BSP, around $\Upsilon_{*}^{K_{s}}=0.2450 \pm 0.0242$. This value is dominated by red giant branch stars.

Regarding the output SSAG-BC03 estimates of $\Upsilon_{*}^{K_{s}}$ for the whole disk, we recover a median $\Upsilon_{*}^{K_{s}}=0.4232$ after BSP iteration number 1 . After iteration number 3 the mean value for the entire disk is $\Upsilon_{*}^{K_{s}}=0.4247 \pm$ 0.0386. For the $\left|\Delta C_{n}\right|<\alpha \sigma_{\mathrm{P}}$ pixels we have $\Upsilon_{*}^{K_{s}}=$ $0.4231 \pm 0.0034$, while for the $\left|\Delta C_{n}\right|>\alpha \sigma_{\mathrm{P}}$ pixels we obtain $\Upsilon_{*}^{K_{s}}=0.4264 \pm 0.0556$, both results after BSP.

Our estimation for $\Upsilon_{*}^{K_{s}}$, derived with MAGPHYSCB07 and SSAG-BC03, are consistent (within 3.0 $\sigma$ ) with the result derived by Just et al. (2015) for the solar cylinder from star counts $\left(\Upsilon_{*}^{K_{s}}=0.34\right)$, and with the average found by Martinsson et al. (2013) for a sample of 30 disk galaxies $\left(\Upsilon_{*}^{K_{s}}=0.31\right)$.

\subsection{Integrated mass estimates}

With respect to the total resolved mass, defined as

$$
M_{*}^{\text {resolved }}=\sum_{j} \sum_{i} M_{* i j},
$$

where $M_{* i j}$ is the stellar mass of the $i^{\text {th }}, j^{\text {th }}$ pixel, we find the following results. By using the MAGPHYSCB07 library we obtain for M 51 a total stellar mass of $M_{*}^{\text {resolved }}=3.84 \times 10^{10} M_{\odot}$ with $\mathrm{ZCR}^{\prime}$, and $M_{*}^{\text {resolved }}=$ $3.22 \times 10^{10} M_{\odot}$ with BSP. The SSAG-BC03 library, meanwhile, leads to $M_{*}^{\text {resolved }}=6.43 \times 10^{10} M_{\odot}$ with $\mathrm{ZCR}^{\prime}$, and $M_{*}^{\text {resolved }}=5.56 \times 10^{10} M_{\odot}$ with BSP. The discrepancy between the SSAG-BC03 and MAGPHYS-CB07 mass estimates is mainly due to the different treatments of the TP-AGB phase (Bruzual 2007). In Figure 15. we show the azimuthally averaged surface mass density vs. radius for M 51 obtained with SSAG-BC03. For most of the disk, the BSP method yields smaller mass estimates than $\mathrm{ZCR}^{\prime}$, resulting in a $\sim 10 \%$ decrease in the total mass. To complement the analysis, we show in Figures $16 \mathrm{a}$ and $16 \mathrm{p}$ (top left and top right panels) the $\Upsilon_{*}^{g}$ maps obtained with the $\mathrm{ZCR}^{\prime}$ method and the BSP algorithm, respectively. Figures $16 \mathrm{c}$ and $16 \mathrm{~d}$ (bottom left and bottom right panels) present the $\Upsilon_{*}^{K_{s}}$ maps from $\mathrm{ZCR}^{\prime}$ and BSP, respectively. Figure 17 shows the azimuthally averaged $\Upsilon_{*}$ for the $g, i$, and $\bar{K}_{s}$ bands, as a function of radius. As expected, the $K_{s}$ profile is virtually constant, while the $g$ and $i$ profiles show variations with radius, with lower values at the outskirts of the disk, as a result of a lower surface brightness and bluer colors (de Jong 1996, Bell \& de Jong 2001).

In Figure 18, we show the azimuthally averaged stellar metallicity, $Z / Z_{\odot}$; similar results are obtained for both BSP and ZCR'. In this figure we also plot the metallicity abundance gradients for M 51 from Moustakas et al. (2010). From ancillary data, Moustakas et al. (2010) estimate radial oxygen abundance gradients for 75 galaxies in the Spitzer Infrared Nearby Galaxies Survey (SINGS, Kennicutt et al. 2003), using both the Kobulnicky \& Kewley (2004; KK04) and the Pilyugin \& Thuan (2005; PT05) calibrations. We transform Moustakas et al. (2010) oxygen abundance gradients in units of $12+\log (\mathrm{O} / \mathrm{H})$, to units of $Z / Z_{\odot}$, adopting (e.g., Martínez-García et al. 2009)

$$
\log \left(Z / Z_{\odot}\right) \simeq 3.12+\log (\mathrm{O} / \mathrm{H}) .
$$

The stellar metallicity we recover with SSAG-BC03 falls between the two curves of Moustakas et al. (2010). Men- 


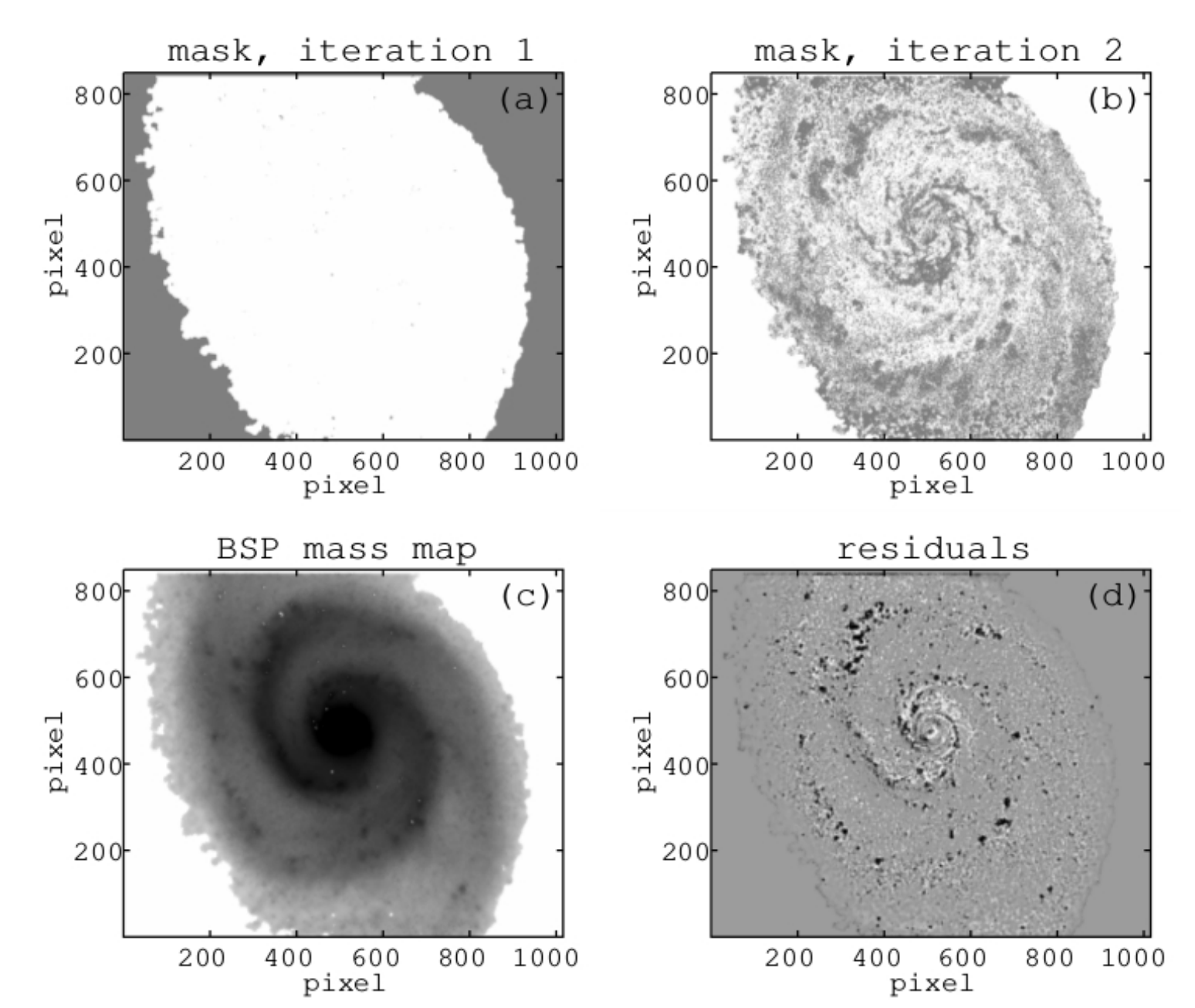

FIG. 11. - Application of the BSP algorithm to the spiral galaxy M 51. The Monte Carlo SPS library used is SSAG-BC03. Top left: resulting mask after iteration number 1. White regions have observed colors within $3 \sigma$ of at least one template in the library. Top right: resulting mask after iteration number 2. Gray regions represent pixels where the assumption of a constant $\Upsilon_{*}^{K_{s}}$ for the whole disk is not fulfilled by the observed colors. Bottom left: resulting mass-map after iteration number 3. Bottom right: residuals after subtracting the mass-map obtained at the end of the BSP algorithm (iteration 3), from a mass-map that assumes a constant $\Upsilon_{*}^{K_{s}}$ (the median after iteration 1). Dark/white regions represent positive/negative mass differences.

tuch Cooper et al. (2012) obtain a similar result for the Whirlpool galaxy, from optical and infrared photometry.

\subsection{Other filter combinations}

In this section we discuss the application of the BSP algorithm with other filter combinations. By using only optical filters, e.g., the $(g-i)$ color and $\Upsilon_{*}^{i}$, the method is not able to recover a spatial structure consistent with the one obtained with optical-NIR combinations. This is due to the fact that the information of the prior spatial structure is missing, as it can only be provided by the NIR bands. The $\Upsilon_{*}^{i}$ cannot be assumed to be constant through the entire disk (see Figure 17); besides, dust lanes can still be noticed near spiral arms, even at the redder optical wavelengths (see Figure 3).

For the case when the $u$ filter is included, we were unable to fit the data satisfactorily. We have quantified the mean $\mathrm{S} / \mathrm{N}$ ratio of the imaging data for the entire disk of M 51 (without applying the Adaptsmooth procedure), and obtain a value of 2.8,23.2, and 29.6 for the $u, g$, and $i$ bands respectively. Taking this into account we can deduce that the issues we encounter when trying to fit the $u$-band SDSS data with our methods are mainly due to their low $\mathrm{S} / \mathrm{N}$ ratio. This shortcoming can be remedied with deeper data. We should also mention that $\Upsilon_{*}$ is more degenerate at shorter wavelengths.

We also applied the BSP algorithm including the Spitzer-IRAC $3.6 \mu \mathrm{m}$ band. We used the colors $(g-i)$ and $(i-3.6 \mu \mathrm{m})$, and $\Upsilon_{*}^{3.6 \mu \mathrm{m}}$. We computed pixel-by-pixel $\sigma_{\text {mag }}$ errors as in section 4 , assuming $\sigma_{\text {calib }} \sim 0.01$ mag for the SDSS images, and $\sigma_{\text {calib }} \sim 0.03 \mathrm{mag}$ for the $3.6 \mu \mathrm{m}$ band (Reach et al. 2005). We corrected for Galactic extinction as in Schlafly \& Finkbeiner (2011), and Chapman et al. (2009). The results with the MAGPHYSBC03 library are shown in Figure 19. It can be noticed that the residuals, i.e., the difference between a massmap that assumes a constant $\Upsilon_{*}^{3.6 \mu \mathrm{m}}$ and the output mass-map from BSP (Figure 19d, bottom right panel), are significantly different from the ones obtained when using the $K_{s}$-band (see Figures $11 \mathrm{~d}$ and $12 \mathrm{~d}$, bottom right panels). We attribute this to polycyclic aromatic hydrocarbons (PAHs) and continuum dust emission at $3.6 \mu \mathrm{m}$. To corroborate this we compare our result to the 

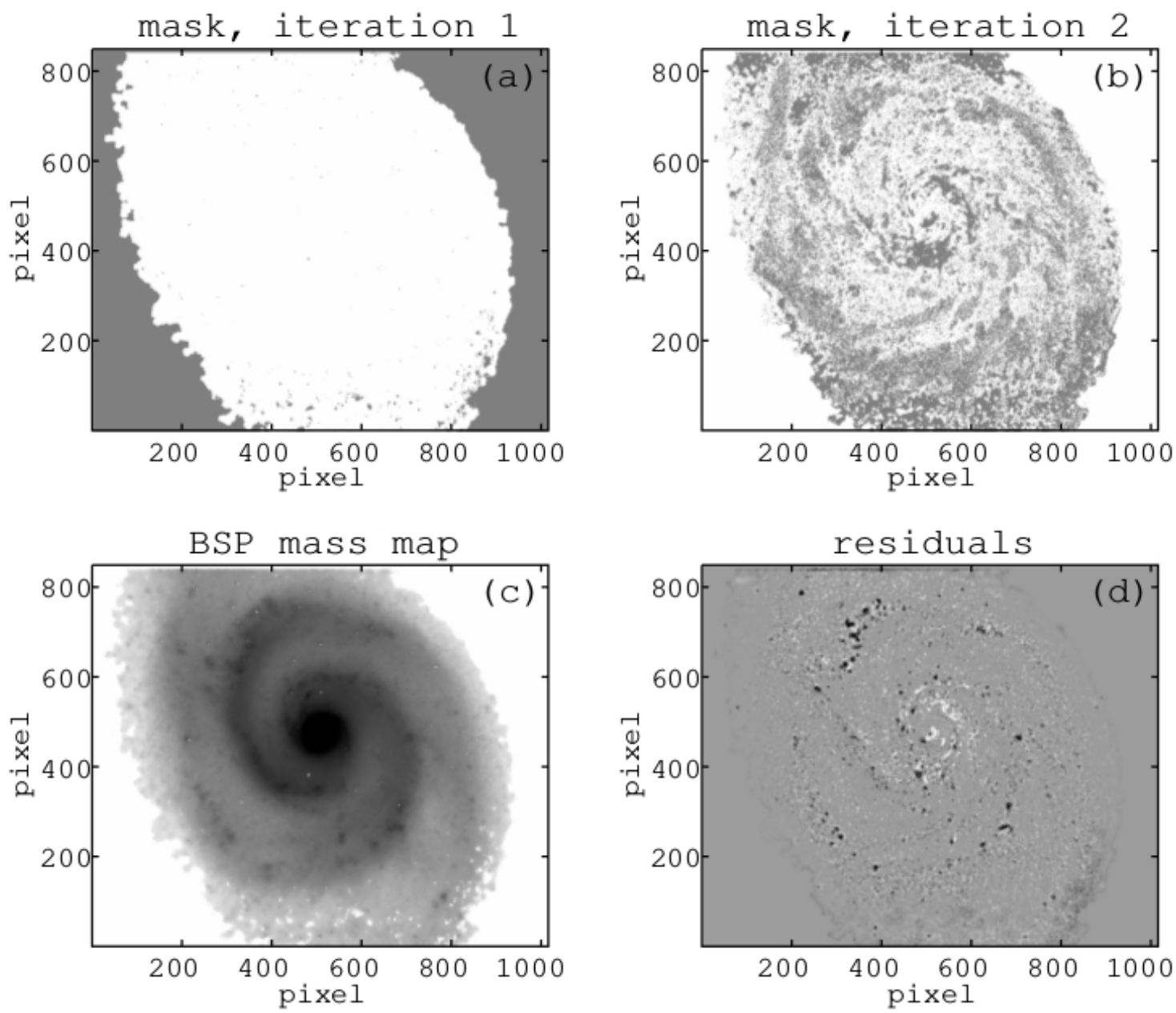

FIG. 12.- Like Figure 11, but for the MAGPHYS-CB07 Monte Carlo SPS library.

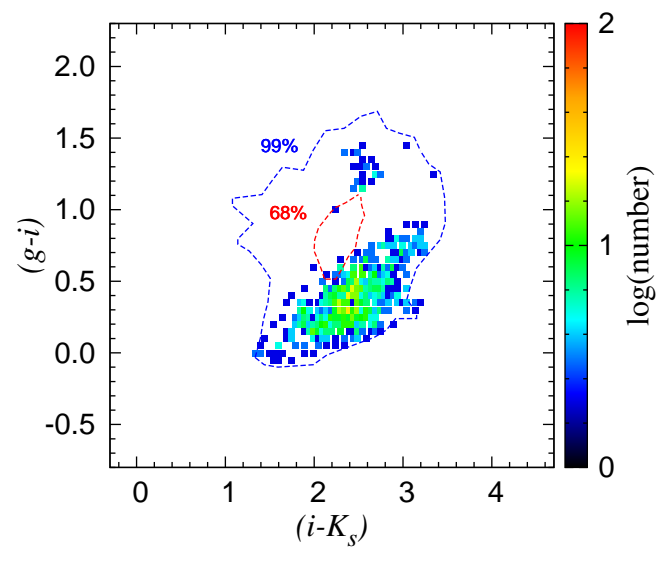

FIG. 13. - 2-D histogram of the observed "positive mass differences" in the residuals (see text), after applying BSP to M 51 (dark regions in Figure 12 $\mathrm{d}$, bottom right panel; see text). The blue/red dashed contour in the plot delimits $99 \% / 68 \%$ of all the observed colors (see Figure 4 left panel).

one derived through the Independent Component Analysis (ICA) method of Meidt et al. (2012, 2014). This method separates the stellar emission from the dust emission; Querejeta et al. (2015) applied it to the Spitzer Survey of Stellar Structure in Galaxies $\left(\mathrm{S}^{4} \mathrm{G}\right.$, Sheth et
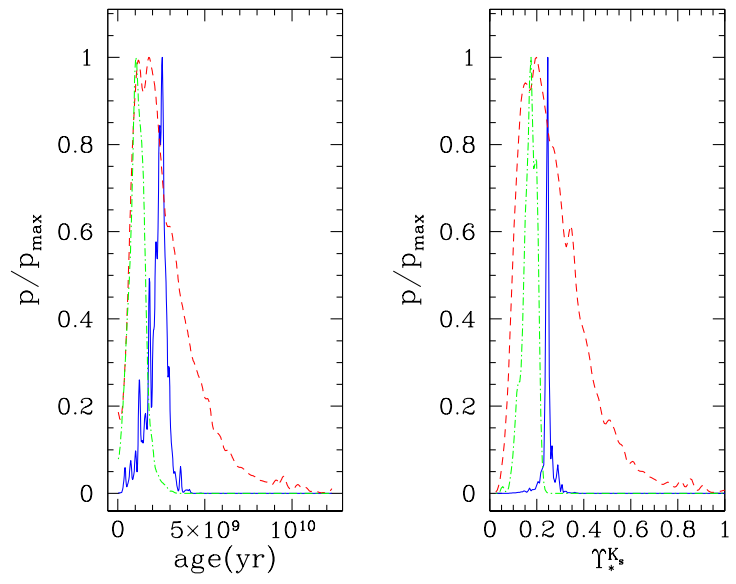

FIG. 14.- M 51 probability $(p)$ distributions with the MAGPHYS-CB07 library. Left: $r$-band light-weighted age (yr); right: mass-to-light ratio $\Upsilon_{*}^{K_{s}}$. Green dashed-dotted line: BSP observed "positive mass differences" in the residuals (see text); blue solid line: $\mathrm{BSP}$ results for the whole disk; red dashed line: $\mathrm{ZCR}^{\prime}$ output for the entire disk.

al. 2010). We compare quantitatively the residuals from BSP with the non-stellar (dust) component from ICA for M 51, by following the same cross-correlation procedure 


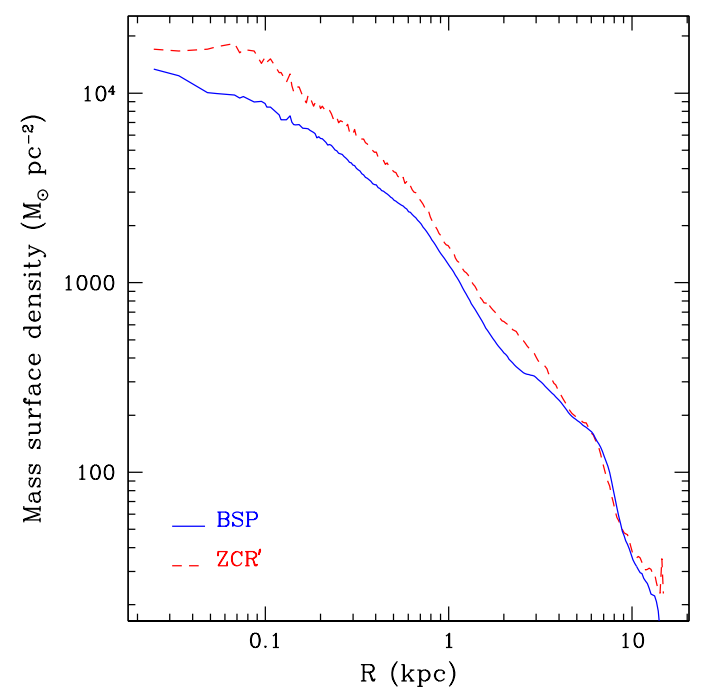

FIG. 15.- Azimuthally averaged mass surface density $\left(M_{\odot}\right.$ $\mathrm{pc}^{-2}$ ) vs. radius, $R$ (kpc), for M 51 with the SSAG-BC03 library. Results are for deprojected mass-maps. Blue solid line: BSP; red dashed line: $\mathrm{ZCR}^{\prime}$

as in section 2.1 (equation 1). The results of this test are shown in Figure 20. We find that there is a strong spatial correlation between the ICA dust component and the $\mathrm{BSP}$ residuals, indicated by the sharp peak at $\theta=0$ in Figure 20. We also compare the $\mathrm{BSP}$ residuals to the stellar component obtained by ICA, and find no spatial correlation at $\theta=0$. Although our adopted SPS library does not include the emission from dust in the $3.6 \mu \mathrm{m}$ band ${ }^{12}$ the BSP algorithm was able to isolate much of it, together with that of red luminous young stars.

A discussion of the differences between ICA and BSP would require further analysis and comparisons using a larger sample of galaxies. This goes beyond the scope of the present work, and will be investigated in a separate publication.

\section{PILOT TEST WITH OTHER GALAXIES}

In order to better understand the differences between using the BSP algorithm of section 3.2 and adopting the $\mathrm{ZCR}^{\prime}$ method (i.e., a maximum likelihood estimate) to obtain resolved maps of stellar mass, we analyzed 90 objects with $H$-band imaging from the Ohio State University Bright Spiral Galaxy Survey (OSUBSGS, Eskridge et al. 2002). The main statistical results from this sample should hold for other surveys, such as SINGS and $\mathrm{S}^{4} \mathrm{G}$. Our sample comprises all objects in the OSUBSGS for which SDSS $g$ and $i$ data are available (see Table 1). A bar chart of the Hubble types of our OSUBSGS sample is shown in Figure 21. We subtracted the $H$-band data "sky offset" (see also Kassin et al. 2006) with either a constant or a plane, depending on the object, and then calibrated the resulting frames with 2MASS. We took optical $g$ and $i$ bands frames from the eighth release (DR8) of the SDSS (Aihara et al. 2011), and mosaicked them

\footnotetext{
12 In principle the emission from dust could be included because it is predicted by MAGPHYS. Nevertheless, the number of templates increases from $5 \times 10^{4}$ to $\sim 6.67 \times 10^{8}$, and CPU time would be $\sim 1 \times 10^{4}$ times larger.
}

with the SWarp software (Bertin 2010). SDSS mosaics were registered and re-sampled to the (lower resolution) $H$-band data with the aid of foreground stars. All foreground stars and background objects were then removed and replaced with random values from the background. The Adaptsmooth code was then used to increase the $\mathrm{S} / \mathrm{N}$ ratio at the outskirts of the disk, while maintaining the relatively higher $\mathrm{S} / \mathrm{N}$ ratio for the inner disk pixels. We adopt a minimum $\mathrm{S} / \mathrm{N}$ ratio per pixel of 10 , and a maximum smoothing radius of 10 .

Together with the OSUBSGS sample, we also analyzed M 51b (companion of M 51, aka NGC 5195) using the same data presented in section 4

\subsection{Mass-maps results}

We adopt the SSAG-BC03 SPS library for all mass estimates for this sample. For simplicity we assume that $\sigma_{\text {mag }} \sim 0.02 \mathrm{mag}$ for every band and pixel. The shortcoming of using a constant $\sigma_{\operatorname{mag}}$ (and consequently a constant $\left.\sigma_{\mathrm{col}}\right)$ for every band and pixel is that some of the fitted values could give slightly $(\sim 0.3 \%$ for individual pixels) different results when compared to the case where individual errors are computed for every pixel. The reason for this is the use of equation 4 together with equation 5 . In our case we adopt two colors, hence equation 4 can be seen as the product of two Gaussian functions (one for each color). In the case where $\sigma_{\text {col }}$ differs for each color, it can be easily demonstrated that this product results in another Gaussian function with different characteristics, including a distinct maximum, when compared to the case of two equal Gaussian functions. Also, the uncertainties in the fitted values will be different. Despite this, the overall results for each object will be practically the same $(\mathrm{a} \sim 0.1 \%$ difference for the resolved total mass estimate).

Some examples of the mass-maps from both the $\mathrm{ZCR}^{\prime}$ approach and BSP are shown in Figure 22. The difference in spatial structures is clearly evident: whereas the $\mathrm{ZCR}^{\prime}$ method gives noisy maps, BSP mass-maps bear a greater similarity to the structures in the NIR-bands. Also shown in this figure are two extreme cases, where dust extinction affects our mass estimates considerably. NGC 7814 is an edge-on spiral with a prominent midplane dust lane. From the first BSP iteration, the colors of the pixels belonging to the dust lanes are identified (and flagged) as outside of the range available in the SPS library. A similar phenomenon occurs with M 51b, since the dust lanes of one of the arms of M 51 are projected directly on it. Consequently, a substantial number of pixels are excluded after the first iteration of BSP. Nevertheless, our recovered stellar mass value for M 51b (see Table 1), obtained via BSP, is $\sim$ half of the one derived for M 51. The same result was obtained by Mentuch Cooper et al. (2012).

As a result of the application of the BSP algorithm to our pilot sample, we identify a trend of the median $\Upsilon_{*}^{H}$ (after BSP iteration number 1) with Hubble type, as predicted by Portinari et al. (2004) and consistent with more recent star formation/more constant SFHs for later Hubble types. A strong linear inverse (or negative) correlation with Hubble type is shown in Figure 23, with a correlation coefficient (Bevington 1969), $r_{x y}=-0.697$ 13

\footnotetext{
13 The value of $r_{x y}$ varies from 0 , for no correlation, to \pm 1 , when
} 

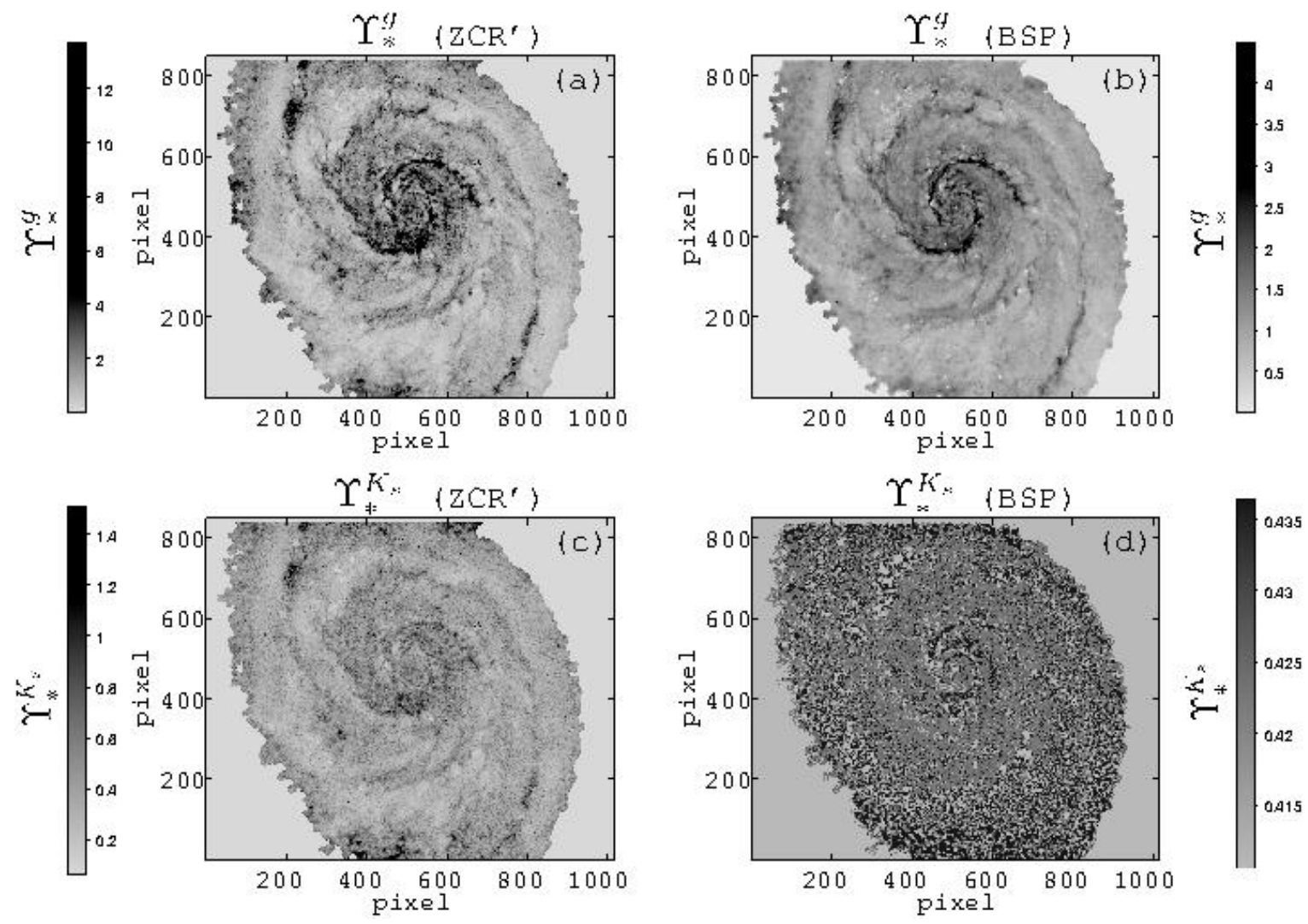

FIG. 16. - $\Upsilon_{*}$ maps for M 51 with the SSAG-BC03 library. Top left: $\Upsilon_{*}^{g}$ obtained with $\mathrm{ZCR}^{\prime}$ method. Top right: $\Upsilon_{*}^{g}$ with BSP algorithm. Bottom left: $\Upsilon_{*}^{K_{s}}, \mathrm{ZCR}^{\prime}$ method. Bottom right: $\Upsilon_{*}^{K_{s}}$, BSP algorithm. Darker pixels indicate higher $\Upsilon_{*}$.

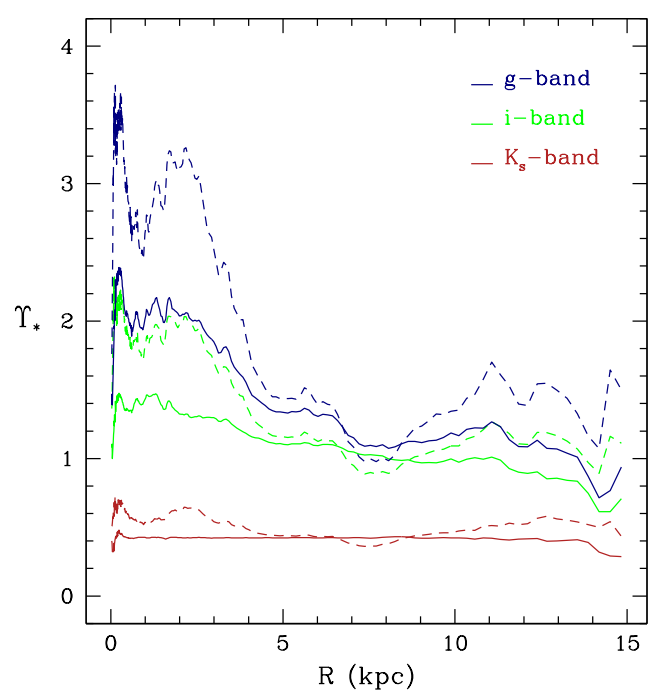

FIG. 17.- Azimuthally averaged $\Upsilon_{*}$ as a function of radius, $R$ (kpc). Solid lines: BSP; dashed lines: ZCR'. Dark blue: $g$-band; green: $i$-band; dark red: $K_{s}$-band. Results are for deprojected maps of M 51 with the SSAG-BC03 library.

Thus, potential biases are introduced when the same $\Upsilon_{*}$ is used for a sample of galaxies with different Hubble types.

there is a full correlation. Generally, $\left|r_{x y}\right| \gtrsim 0.7$ is considered a strong correlation, $\left|r_{x y}\right| \approx 0.5$ a moderate correlation, and $\left|r_{x y}\right| \approx$ 0.3 a weak correlation.

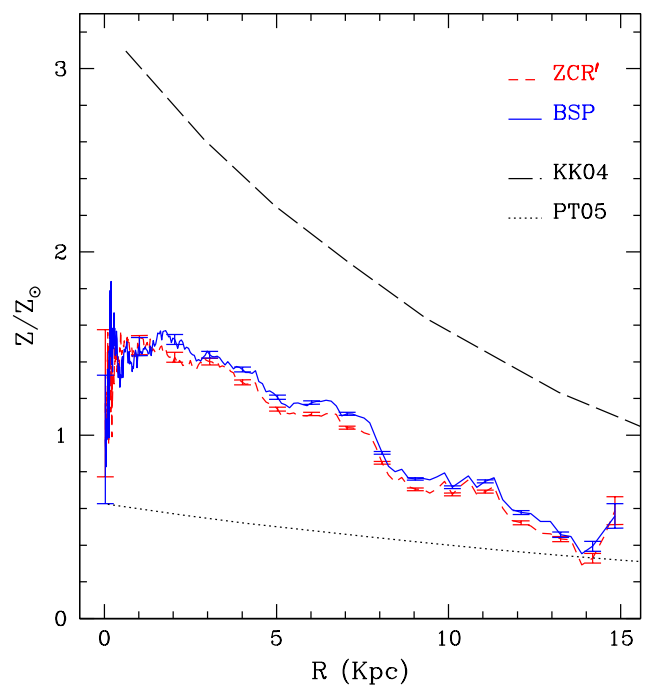

FIG. 18.- Azimuthally averaged stellar metallicity, $Z / Z_{\odot}$. Blue solid line: $\mathrm{BSP}$; red dashed line: $\mathrm{ZCR}^{\prime}$. Results are for deprojected maps of M 51 with the SSAG-BC03 library. For comparison we show the metallicity abundance gradients of Moustakas et al. (2010), using the KK04 (black dashed line) and PT05 (black dotted line) calibrations.

The total resolved stellar masses, $M_{*}^{\text {resolved }}$ (equation 15), obtained, respectively, with the BSP algorithm, $M_{*}^{\mathrm{BSP}}$, and with the $\mathrm{ZCR}^{\prime}$ approach, $M_{*}^{\mathrm{ZCR}}$, are given in Table 1. In Figure 24 we display the behavior of the 

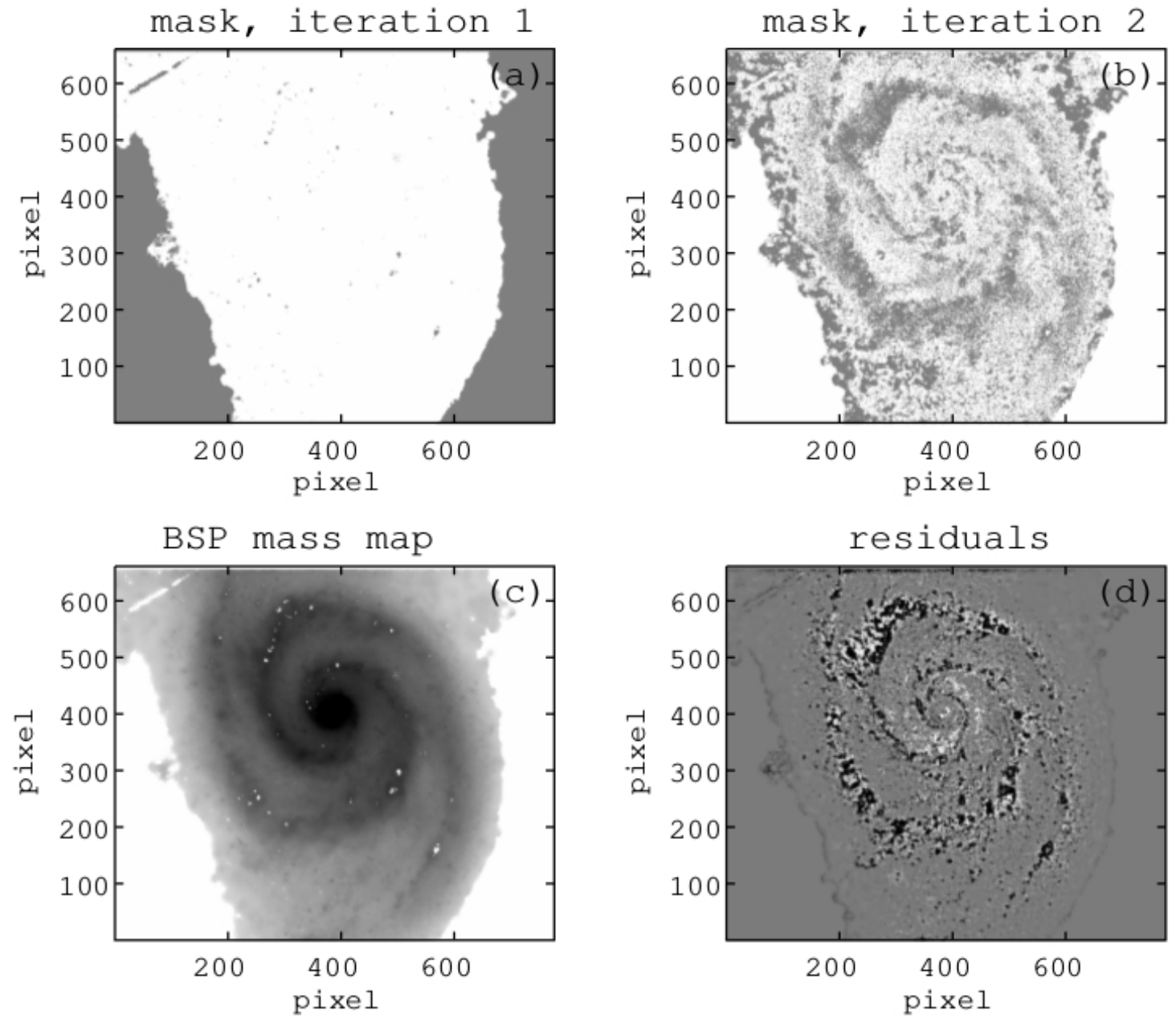

FIG. 19. - Application of BSP algorithm to M 51 with $(g-i),(i-3.6 \mu \mathrm{m}), \Upsilon_{*}^{3.6 \mu \mathrm{m}}$, and the MAGPHYS-BC03 SPS library. Panels organized as in Figures 11 and 12

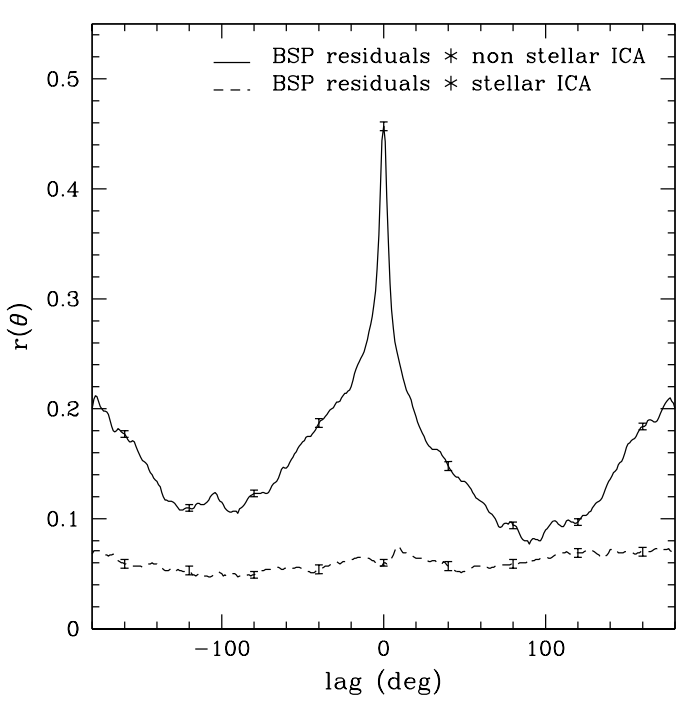

FIG. 20.- Cross-correlation functions (see text) for BSP residuals using the $3.6 \mu \mathrm{m}$-band. Solid line: with the non-stellar (dust) emission from ICA (Meidt et al. 2012, 2014 Querejeta et al.|2015); the absolute maximum is at $\theta=0^{\circ}$. Dashed line: with the stellar emission from ICA. Height of error bars is $2 \sigma_{c c}$.

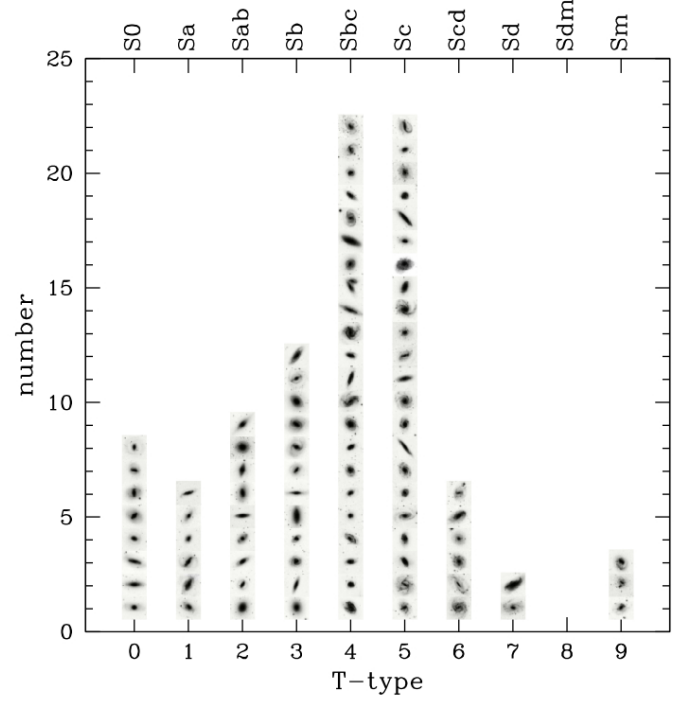

FiG. 21. - Bar chart of Hubble types for our galaxy sample (90 objects). Embedded images from the Digitized Sky Survey, DSS (blue).

ratio $M_{*}^{\mathrm{BSP}} / M_{*}^{\mathrm{ZCR}}$ vs. $M_{*}^{\mathrm{BSP}}$. From these data we find that BSP mass estimates are on average $\sim 10 \%$ lower 

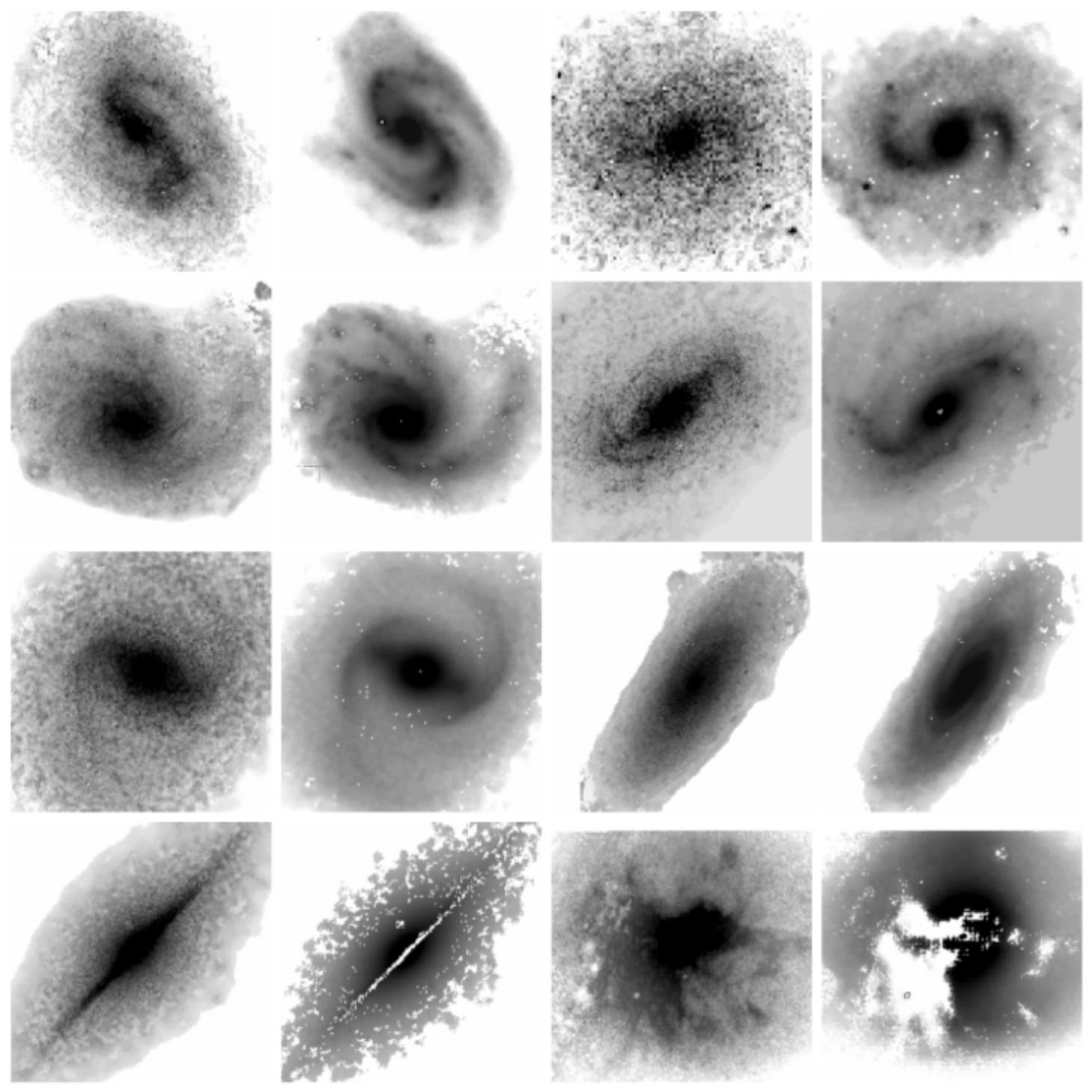

FIG. 22. - Resolved maps of stellar mass. Columns 1 and 3: ZCR'; columns 2 and 4: BSP. From left to right, in pairs: NGC 157, NGC 1042, NGC 4254, NGC 4051, NGC 4548, NGC 7606, NGC 7814, and M 51b.

than those derived from $\mathrm{ZCR}^{\prime}$, similarly to the $\mathrm{M} 51$ result. We also investigate possible trends of the ratio $M_{*}^{\mathrm{BSP}} / M_{*}^{\mathrm{ZCR}}$ with Hubble Type; with the ratio of major to minor galaxy axes $a / b$; with star formation rate, $\Psi$; and with $V$-band optical depth, $\tau_{V}$. We find no strong or moderate correlations with these parameters, except for the star formation rate, having $r_{x y}=-0.061$ for Hubble Type, $r_{x y}=-0.297$ for galaxy axial ratio (excluding the edge-on object NGC 7814), and $r_{x y}=0.082$ for the median $\tau_{V}$ for the entire disk, obtained via BSP. We computed the star formation rate averaged over the last $10^{8}$ yr from the parameters of the fitted templates as

$$
\langle\Psi\rangle=\frac{\int_{t-t_{\text {last }}}^{t} \Psi\left(t^{\prime}\right) \mathrm{d} t^{\prime}}{t_{\text {last }}}
$$

where time $t$ corresponds to the current $\Psi$, and $t_{\text {last }}=$ $10^{8}$ yr. We calculate $\langle\Psi\rangle$ on a pixel-by-pixel basis and then sum over all pixels (in the same way as the resolved mass estimate). We also estimate the specific star formation rate averaged over the last $10^{8} \mathrm{yr}$ :

$$
\langle\Psi\rangle_{\mathrm{S}}=\frac{\int_{t-t_{\text {last }}}^{t} \frac{\Psi\left(t^{\prime}\right)}{M_{*}\left(t^{\prime}\right)} \mathrm{d} t^{\prime}}{t_{\text {last }}} \approx\langle\Psi\rangle M_{*}^{-1},
$$

where $M_{*}$ is the current stellar mass. In this manner we obtain the resolved $\langle\Psi\rangle$, and $\langle\Psi\rangle_{\mathrm{S}}$, for the corresponding object. In Figure 25 we show the ratio $M_{*}^{\mathrm{BSP}} / M_{*}^{\mathrm{ZCR}}$ vs. the resolved $\left\langle\Psi^{\mathrm{BSP}}\right\rangle_{\mathrm{S}}$ for the whole disk. The correlation coefficient is $r_{x y}=-0.335$ indicating a weak inverse correlation. In the case of the resolved $\langle\Psi\rangle$ we obtain a correlation coefficient of $r_{x y}=-0.240$. These results suggest that the bias in the resolved mass values $M_{*}^{\mathrm{ZCR}}$, when compared to $M_{*}^{\mathrm{BSP}}$, is weakly related to the star formation rate over the disk.

For completeness, we show in Figure 26 the resolved galaxy "main sequence" of star formation (see e.g., Noeske et al. 2007, Daddi et al. |2007, Elbaz et al. 2007. 


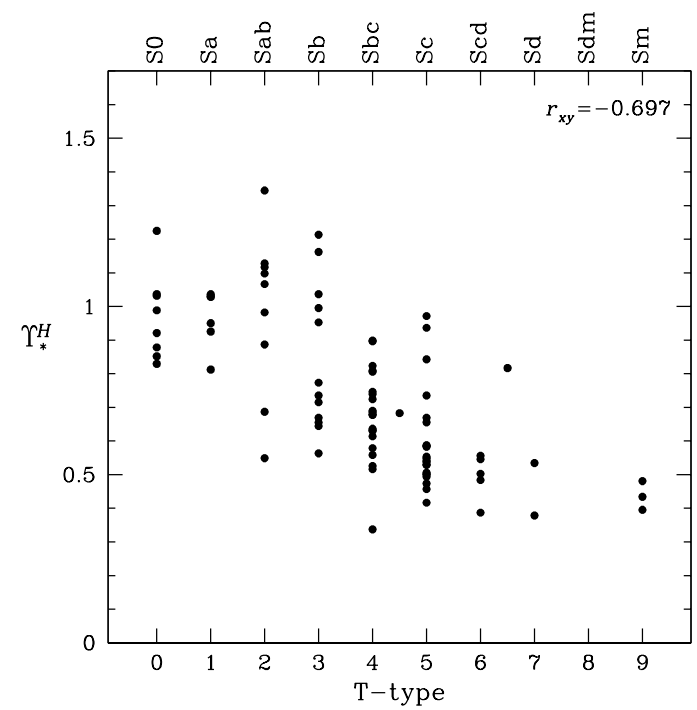

FIG. 23.- Median $\Upsilon_{*}^{H}$ vs. Hubble T-type after BSP iteration number 1 , applied to the OSUBSGS pilot sample. Although with some scatter, a strong $\left(r_{x y}=-0.697\right)$ trend is observed whereby $\Upsilon_{*}^{H}$ decreases with increasing $\mathrm{T}$ (later Hubble type).

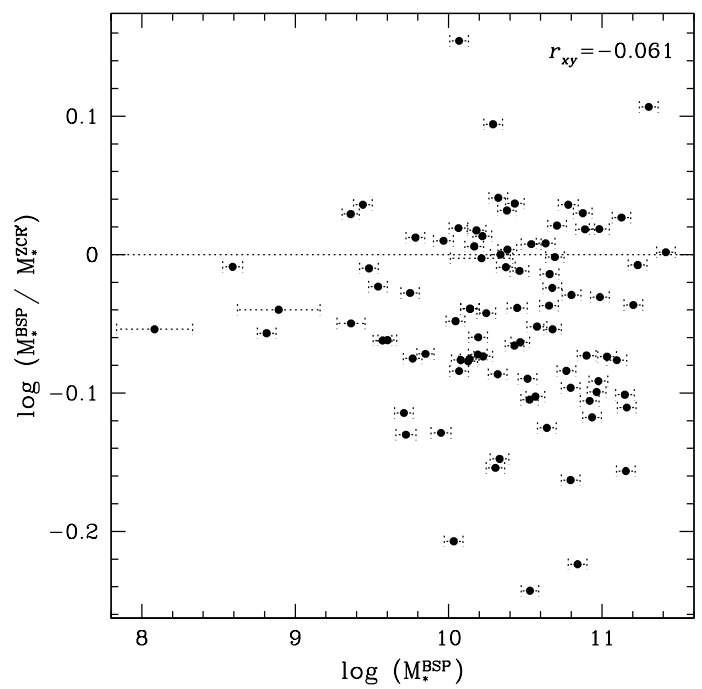

FIG. 24.- Comparison of total resolved stellar mass estimates, $\log \left(M_{*}^{\mathrm{BSP}} / M_{*}^{\mathrm{ZCR}}\right)$ vs. $\log \left(M_{*}^{\mathrm{BSP}}\right)$. Horizontal error bars for $M_{*}^{\mathrm{BSP}}$ represent the propagated uncertainty in the distance to the objects.

Salim et al. 2007), i.e., the relationship between resolved $\langle\Psi\rangle$ and $M_{*}^{\text {resolved. }}$. We find that this correlation is stronger with BSP $\left(r_{x y}=0.853\right)$ when compared to $\mathrm{ZCR}^{\prime}\left(r_{x y}=0.797\right)$.

\subsection{Comparison with unresolved mass estimates}

We also obtain for each object an unresolved mass estimate, $M_{*}^{\text {unresolved }}$. To this end, we fit the global $(g-i)$ and $\left(i-H^{*}\right)$ colors of the object to all templates, and get the optimum one via equation 4 Global magnitudes are calculated by summing the intensities of all the pixels:

$$
\operatorname{mag}^{\text {global }}=-2.5 \log _{10} \sum_{j} \sum_{i} f_{i j}+z p,
$$

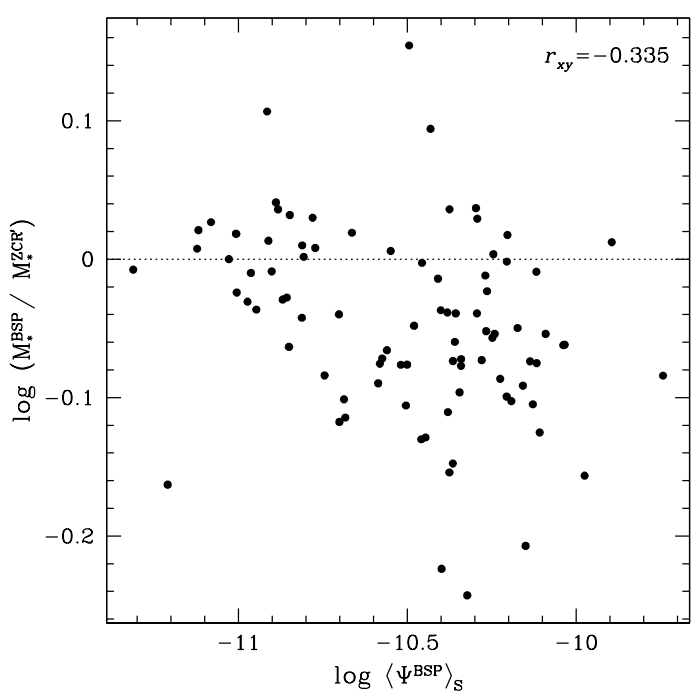

FIG. 25.- Decimal logarithm of $\left(M_{*}^{\mathrm{BSP}} / M_{*}^{\mathrm{ZCR}}\right) \quad$ vs. $\log \left\langle\Psi^{\mathrm{BSP}}\right\rangle_{\mathrm{S}}$, with $\Psi_{\mathrm{S}}$ in $\mathrm{yr}^{-1}$. The resolved specific star formation rate is obtained as the sum of all pixels in the disk using BSP. A weak correlation is observed with negative correlation coefficient $r_{x y}=-0.335$.
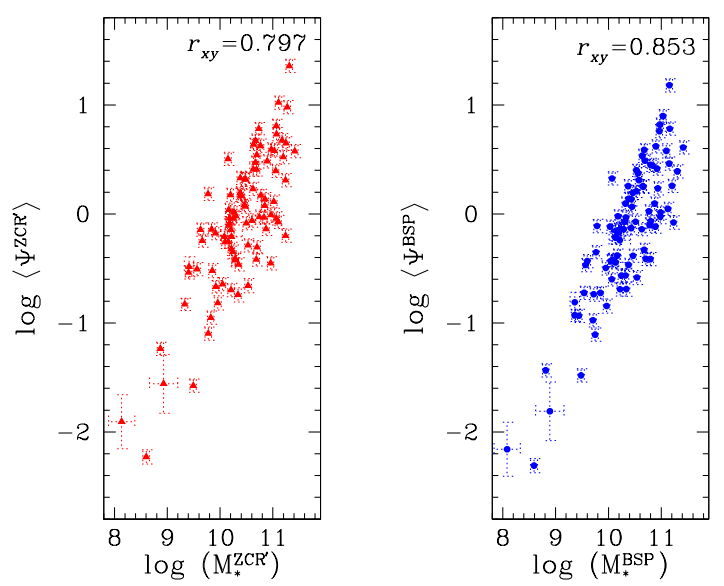

FIG. 26. - The resolved "main sequence" of star forming galaxies for the OSUBSGS pilot sample with the SSAG-BC03 library. Left panel (red triangles): $\mathrm{ZCR}^{\prime}$; right panel (blue dots): BSP. Resolved star formation rate, $\langle\Psi\rangle$, in units of $M_{\odot} \mathrm{yr}^{-1}$, and resolved stellar mass, $M_{*}^{\text {resolved }}$, in units of $M_{\odot}$.

where $f_{i j}$ is the intensity of the $i^{\text {th }}, j^{\text {th }}$ pixel at a certain band, and $z p$ is the appropriate zero point. The same number of pixels is used in all mass estimates for the same object.

We compare in Figure $27 M_{*}^{\text {unresolved }}$ with $M_{*}^{\text {resolved }}$. The results for $\mathrm{ZCR}^{\prime}$ are shown in the left panel, and those for BSP are presented on the right. On average we find that, for our sample of galaxies, unresolved values underestimate masses by $\sim 20 \%$ compared to $\mathrm{ZCR}^{\prime}$, but only by $\sim 10 \%$ relative to BSP. We also find, however, that for a fraction of the objects $(15 \%$ when comparing to $\mathrm{ZCR}^{\prime}$ and $25 \%$ vis-à-vis BSP) the unresolved mass estimates are actually larger than those determined from resolved studies. The estimate we can get for an unresolved mass depends on how each pixel contributes to the global colors. Pixels that contain relatively young star forming 

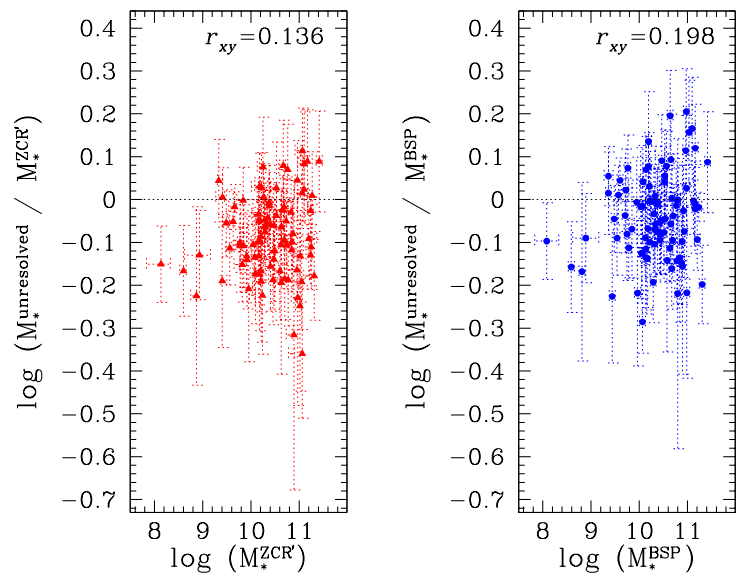

FIG. 27.- Comparison of total unresolved and resolved stellar mass estimates. Left panel (red triangles): $\mathrm{ZCR}^{\prime}$; right panel (blue dots): BSP.
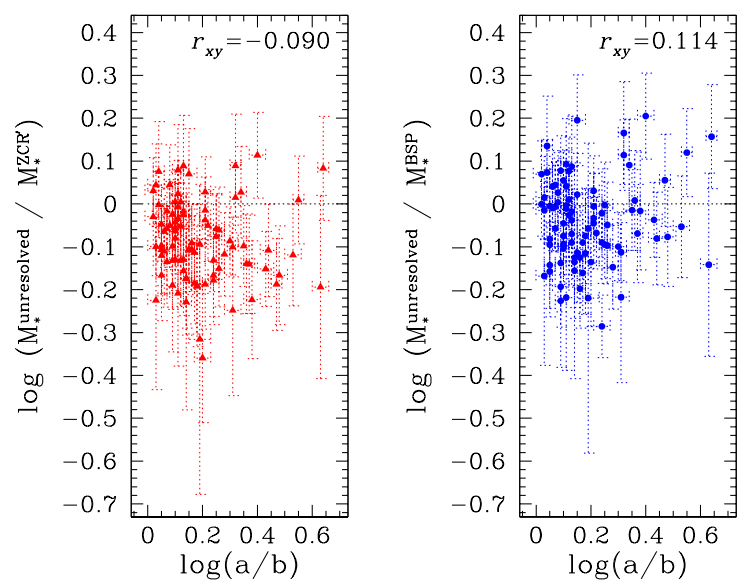

FIG. 28.- Ratio of unresolved to resolved stellar mass estimates vs. galaxy axial ratio, $a / b$ (from RC3, de Vaucouleurs et al. 1991). Left panel (red triangles): $\mathrm{ZCR}^{\prime}$; right panel (blue dots): BSP.

regions will lead to global bluer colors, and consequently a lower global $\Upsilon_{*}$ (see Figure 1 or 6). On the other hand, pixels that contain extinction regions, due to dust, will lead to global redder colors and therefore a higher global $\Upsilon_{*}$. In spite of these possible effects the error bars for $\log \left(M_{*}^{\text {unresolved }} / M_{*}^{\text {resolved }}\right)>0$ (see Figure 27) are within the $\log \left(M_{*}^{\text {unresolved }} / M_{*}^{\text {resolved }}\right) \sim 0$ value.

We find no correlation of $M_{*}^{\text {unresolved }} / M_{*}^{\text {resolved }}$ with Hubble type $\left(r_{x y}=0.064\right.$ for BSP, and $r_{x y}=0.024$ for $\left.\mathrm{ZCR}^{\prime}\right)$, global $(g-i)$ color $\left(r_{x y}=0.057\right.$ for BSP, $r_{x y}=-0.025$ for $\left.\mathrm{ZCR}^{\prime}\right)$, or median $\tau_{V}\left(r_{x y}=0.055\right.$ for BSP, $r_{x y}=0.116$ for $\mathrm{ZCR}^{\prime}$ ). The correlation test was also negative for galaxy inclination (see Figure 28), with $r_{x y}=0.114$ for BSP, and $r_{x y}=-0.090$ for ZCR'. When comparing the resolved $\langle\Psi\rangle_{\mathrm{S}}$ for each object with the ratio $M_{*}^{\text {unresolved }} / M_{*}^{\text {resolved }}$, we find a weak positive correlation $\left(r_{x y}=0.262\right)$ for BSP, and no correlation $\left(r_{x y}=0.118\right)$ for $\mathrm{ZCR}^{\prime}$. In Figure 29 we show the ratio $M_{*}^{\text {unresolved }} / M_{*}^{\text {resolved }}$ vs. resolved $\langle\Psi\rangle$. The correlation coefficients are $r_{x y}=0.336$ for BSP (right panel), and $r_{x y}=0.212$ for $\mathrm{ZCR}^{\prime}$ (left panel) indicating a weak correlation in our case test.
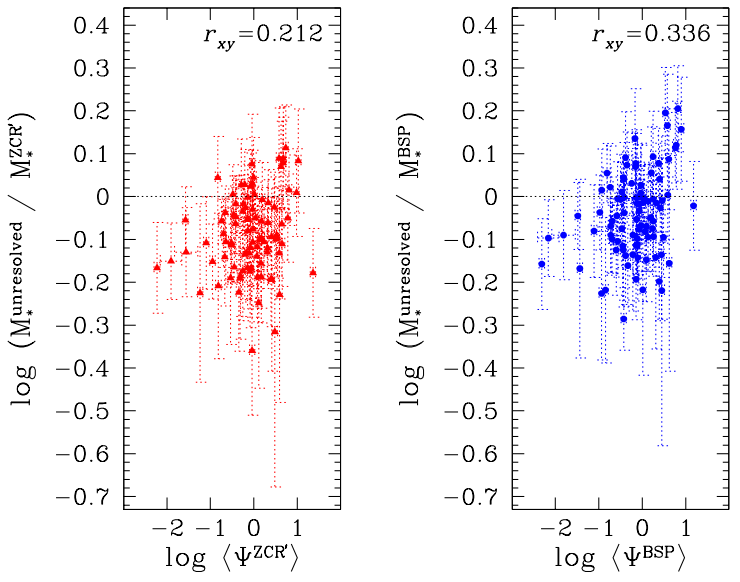

FIG. 29.- Ratio of unresolved to resolved stellar mass estimates vs. resolved star formation rate $\langle\Psi\rangle\left(M_{\odot} \mathrm{yr}^{-1}\right)$. Left panel (red triangles): $\mathrm{ZCR}^{\prime}$; right panel (blue dots): $\mathrm{BSP}$.

\section{UNCERTAINTIES IN THE STELLAR MASS ESTIMATES}

All the stellar mass estimates given in Table 1 are for the SSAG-BC03 library; if, instead, the MAGPHYSCB07 library is used, the masses will be smaller $(\sim 50 \%)$, due to the different treatment of the TP-AGB. Hence, the dominant source of error is systematic.

Regarding the uncertainty in the mass per pixel, we obtain a mean value for the entire disk of $\sim 27 \%$ with $\mathrm{ZCR}^{\prime}$, and of $\sim 3 \%$ with BSP. The reduction of the uncertainty in BSP is due to the inclusion of equation 7 in the calculations. The random errors in the total resolved mass estimates, on the other hand, are rather small, given the very large number of pixels involved in the calculations $\left(\sim 6 \times 10^{5}\right.$ and $\sim 2 \times 10^{4}$ pixels, for M 51 and the OSUBSGS objects, respectively). For an object with $n_{\text {pix }}$ pixels the relative uncertainty $\left(\sigma_{\text {mass }} /\right.$ mass $)$ decreases as $\sim \frac{1}{\sqrt{n_{\text {pix }}}}$. Hence the random uncertainties in the total resolved mass estimates tend to be less than $0.1 \%$. The random uncertainties in the median $\Upsilon_{*}$ (after iteration number 1 ), and $\langle\Psi\rangle$ are also relatively small due to the large number of pixels involved in the calculations. With regard to the systematic uncertainty due to the zero point error $\sigma_{\text {calib }}$, we estimate a $3 \%$ relative error in the resolved mass estimates, and the median $\Upsilon_{*}$. However, this systematic error dominates the relative uncertainties in $M_{*}^{\text {unresolved }}$ (see equation 19), which have a median of $\sim 22 \%$ (see Table 1 ).

Another source of systematic error is the uncertainty in the distance to the objects, $\sigma_{\text {dist }}$. Propagating $\sigma_{\text {dist }}$ leads to $\mathrm{a} \sim 14 \%$ uncertainty in the mass, and $\langle\Psi\rangle$, for all galaxies in our pilot sample, with the exception of NGC 3319, NGC 4051, and NGC 4212, for which the uncertainty in the mass is $\sim 55 \%$. However, the contribution of this uncertainty is negligible for the mass ratio of any single galaxy $\left(M_{*}^{\mathrm{BSP}} / M_{*}^{\mathrm{ZCR}}\right.$ or $\left.M_{*}^{\text {unresolved }} / M_{*}^{\text {resolved }}\right)$, since all mass estimates are equally affected. Equivalently, $\langle\Psi\rangle_{\mathrm{S}}$ is not affected by $\sigma_{\text {dist }}$.

Regarding the choice of the IMF, our default is Chabrier (2003). Stellar masses can be $\sim 1.7 \pm 0.3$ 
times larger with the Salpeter (1955) IMF, and $\sim 1.1 \pm$ 0.03 times larger with the Kroupa (2001) IMF.

We also have quantified that using only a constant $\Upsilon_{*}$ (i.e., skipping iteration number 3) yields masses per pixel $\sim 1 \%$ higher on average, and up to $\sim 30 \%$ larger in localized regions.

\subsection{Dependence on disk inclination}

Stellar mass is an intrinsic property of galaxies, independent of inclination to the line of sight. Stellar mass determinations from broad-band colors, however, are independent of inclination only as surface brightness at different wavelengths is independent of it. Maller et al. (2009) study the effects of inclination on mass estimates, by comparing a statistically significant sample of edge-on $(a / b \geq 3.33)$ and face-on $(a / b \leq 1.18)$ SDSS galaxies. They find no statistical difference for masses derived from $K$-band photometry by Bell et al. (2003) but, on the other hand, point out the very important corrections with inclination that are necessary for the $B$-band (Driver et al. 2007).

We remind the reader that all our calculations are based on the effective $\Upsilon_{*}$. Extinction effects may introduce biases with inclination. In subsequent publications we will address this issue in more detail.

\section{CONCLUSIONS}

We have demonstrated quantitatively that resolved maps of stellar mass obtained by the maximum likelihood estimate (as in ZCR) yield biased spatial structures. The bias consists in a filamentary morphology, and a spatial coincidence between dust lanes and purported stellar mass surface density. The bias is due to a limited $\Upsilon_{*}$ accuracy $(\sim 0.1-0.15$ dex $)$ arising from uncertainties inherent to observations, and to degeneracies between templates of similar colors in the SPS libraries. Similar observed colors will yield the mode $\Upsilon_{*}$. Here, we have succeeded in mitigating the bias with the BSP algorithm we have developed. We have applied the new algorithm to M 51 and a pilot sample of 90 spirals. BSP effectively identifies and isolates the old stellar population, and the output mass-maps bear more resemblance to NIR structures.

The results also indicate that total resolved mass estimates obtained by adding up the pixel-by-pixel contributions are on average $\sim 10 \%$ lower with BSP than with the $\mathrm{ZCR}^{\prime}$ approach. Hence, unresolved stellar mass estimates for our pilot sample underestimate the mass by $\sim 20 \%$ when compared to the resolved $\mathrm{ZCR}^{\prime}$ results, but only by $\sim 10 \%$ vis-à-vis BSP.

The fact that the same SPS libraries can produce, or not, filamentary structures where the mass is supposedly organized indicates that such structures are merely an artifact of the method, and not real massive features present in disk galaxies.

An additional advantage of using a spatial structure prior for mass estimates is its independence of SPS model parameters (e.g., SFH, metallicity, dust, age, etc.) or ingredients (e.g., TP-AGB phase, or IMF). Galaxy masses determined from SPS models should be compared to results of independent studies, e.g., the Disk Mass Sur-

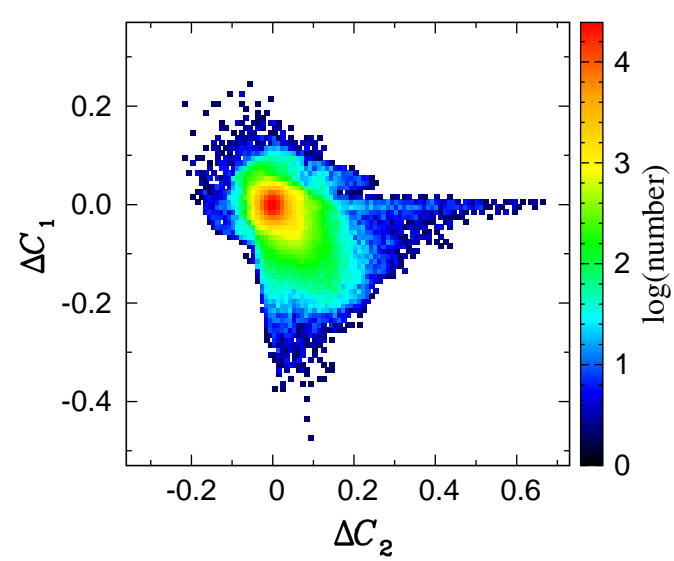

FIG. 30.- 2-D histogram of the $\Delta C_{1}=(g-i)^{\text {obs }}-(g-i)^{\text {template }}$, and $\Delta C_{2}=\left(i-K_{s}\right)^{\text {obs }}-\left(i-K_{s}\right)^{\text {template }}$ distributions before applying the $\left|\Delta C_{n}\right|<\alpha \sigma_{\mathrm{P}}$ condition at iteration number 2 of BSP. Data pixels for M 51, adopting the MAGPHYS-CB07 library.

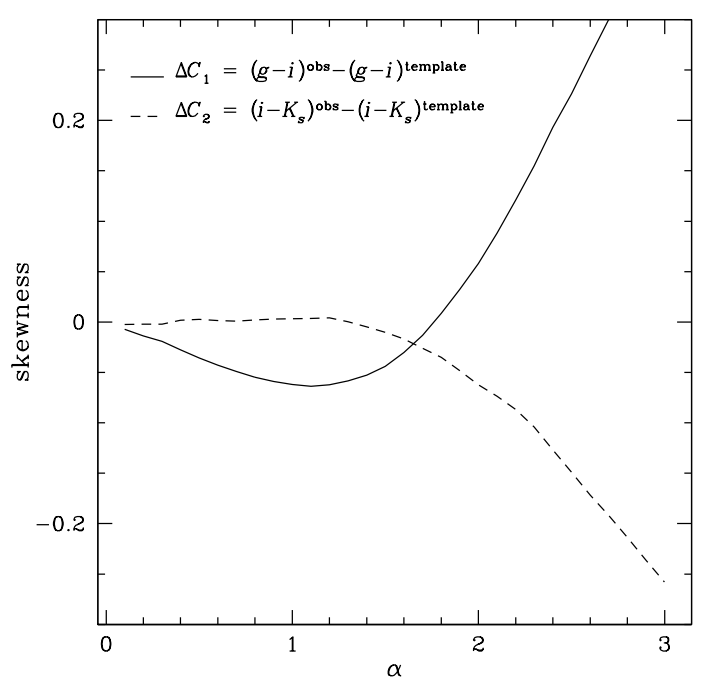

FIG. 31.- "Skewness curves" of the $\Delta C_{1}$ and $\Delta C_{2}$ distributions after applying the $\left|\Delta C_{n}\right|<\alpha \sigma_{\mathrm{P}}$ condition for different $\alpha$ values. Same data as in Figure 30

vey (DMS, Bershady et al. 2010 ${ }^{14}$. Systematic uncertainties may be constrained through these comparisons (see also de Jong \& Bell 2007).

We acknowledge the referee for her/his comments and suggestions that significantly improved the quality of the manuscript. We appreciate discussions with and comments from Margarita Rosado, Ivânio Puerari, Bernardo Cervantes-Sodi, Sebastián Sánchez, Fabián Rosales-Ortega, Olga Vega, Edgar Ramírez, and William Wall. We thank Alfredo Mejía-Narváez for useful discussions about the SSAG parameters.

EMG acknowledges support from INAOE during the initial stages of this research, and from IRyA during the development of the project; he gives special thanks to his

14 DMS uses measurements of the vertical velocity dispersion of disk stars as a dynamical constraint on the mass surface density of spiral disks. 
mother, Gilda García.

RAGL acknowledges the support from DGAPA, UNAM, through project PAPIIT IG100913, and from CONACyT, Mexico, through project SEP-CONACyT I0017-151671.

GMC acknowledges support from IRyA during the initial stages of this work.

GBA acknowledges support for this work from the National Autonomous University of Mexico (UNAM), through grant PAPIIT IG100115.

Funding for SDSS-III has been provided by the Alfred P. Sloan Foundation, the Participating Institutions, the National Science Foundation, and the U.S. Department of Energy Office of Science. The SDSS-III web site is http://www.sdss3.org/. SDSS-III is managed by the Astrophysical Research Consortium for the Participating Institutions of the SDSS-III Collaboration including the University of Arizona, the Brazilian Participation Group, Brookhaven National Laboratory, Carnegie Mellon University, University of Florida, the French Participation Group, the German Participation Group, Harvard University, the Instituto de Astrofísica de Canarias, the Michigan State/Notre Dame/JINA Participation Group, Johns Hopkins University, Lawrence Berkeley National Laboratory, Max Planck Institute for Astrophysics, Max
Planck Institute for Extraterrestrial Physics, New Mexico State University, New York University, Ohio State University, Pennsylvania State University, University of Portsmouth, Princeton University, the Spanish Participation Group, University of Tokyo, University of Utah, Vanderbilt University, University of Virginia, University of Washington, and Yale University.

This work made use of data from the Ohio State University Bright Spiral Galaxy Survey, which was funded by grants AST-9217716 and AST-9617006 from the United States National Science Foundation, with additional support from the Ohio State University.

This research has made use of the NASA/IPAC Extragalactic Database (NED) which is operated by the Jet Propulsion Laboratory, California Institute of Technology, under contract with the National Aeronautics and Space Administration.

The Digitized Sky Surveys were produced at the Space Telescope Science Institute (STScI) under U.S. Government grant NAG W-2166. DSS images can be found at the URL https://archive.stsci.edu/cgi-bin/dss_ form.

This work has made use of the adaptive smoothing code Adaptsmooth, developed by Stefano Zibetti and available at the URL http://www.arcetri.astro.it/ zibetti/ Software/ADAPTSMOOTH.html.

\section{APPENDIX}

\section{DETERMINATION OF THE $\alpha$ PARAMETER FOR BSP.}

The last step of iteration number 2 is to identify the pixels which satisfy the condition $\left|\Delta C_{n}\right|<\alpha \sigma_{\mathrm{P}}$. From the definition of $\Delta C_{n}=C_{n}^{\text {obs }}-C_{n}^{\text {template }}$, we have:

$$
\Delta C_{1}=(g-i)^{\mathrm{obs}}-(g-i)^{\text {template }},
$$

and

$$
\Delta C_{2}=\left(i-K_{s}\right)^{\text {obs }}-\left(i-K_{s}\right)^{\text {template }},
$$

for the $(g-i)$, and $\left(i-K_{s}\right)$ colors, respectively. The value of $\sigma_{\mathrm{P}}$ is computed from equation 12 In Figure 30 we show a plot of $\Delta C_{1}$ vs. $\Delta C_{2}$ for the case of the MAGPHYS-CB07 SPS library, before applying the $\left|\Delta C_{n}\right|<\alpha \sigma_{\mathrm{P}}$ condition to the pixels of M 51 (see section 4). From these $\Delta C_{n}$ distributions we obtain $\sigma_{\mathrm{P}}=0.02376$ and $\sigma_{\mathrm{P}}=0.02723$, for the $(g-i)$ and $\left(i-K_{s}\right)$ colors, respectively. The purpose of applying the $\left|\Delta C_{n}\right|<\alpha \sigma_{\mathrm{P}}$ condition is to isolate the pixels that deviate significantly from the value $\Delta C_{n} \sim 0$. In Figure 31 we show a plot of the skewness (a measure of the degree of asymmetry) of the $\Delta C_{1}$ and the $\Delta C_{2}$ distributions, after applying the $\left|\Delta C_{n}\right|<\alpha \sigma_{\mathrm{P}}$ condition for different $\alpha$ values. The "skewness curves" have extrema near $\alpha \sim 1$, a minimum for the $\Delta C_{1}$ curve and a maximum for the $\Delta C_{2}$ curve. These extrema values indicate a transition of the shape of the $\Delta C_{n}$ distributions. Similar plots are obtained for the MAGPHYS-BC03 and the SSAG-BC03 libraries, which also have extrema near $\alpha \sim 1$. The $\Delta C_{n}$ distributions become extremely asymmetric for $\alpha>1$. Therefore, in a statistical manner, applying the condition $\left|\Delta C_{n}\right|<\alpha \sigma_{\mathrm{P}}$, with $\alpha \sim 1$, fulfills our purposes.

\section{PROBABILITY DISTRIBUTION FUNCTIONS FOR DISK PARAMETERS.}

In this section we explain the method we use to obtain the probability distributions for the disk parameters shown in Figure 14 After applying either ZCR $\mathrm{ZCR}^{\prime}$ or BSP to a given object, we obtain a set of templates which were fitted to a group of pixels (e.g., for the whole disk we use the pixels shown in Figure 12 a, top left panel). We then use a Gaussian kernel density method (Keen 2010) to estimate the probability density function. In essence, the kernel method produces a smoothed version of a histogram. First, we build a grid for each parameter (e.g., age) within the range of values given by the SPS library. The grid contains 512 bins and has a distinct bin width of size $b_{w}^{\mathrm{par}}$ for each parameter. A single parameter has bins of equal width, $b_{w}^{\mathrm{par}}$, which is estimated as the difference between the highest value minus the lowest value, divided by 512 . Then we count how many pixels fall into each bin, i.e., we build a histogram of the pixel population given a certain parameter. We then calculate, for each histogram, a smoothing parameter called the bandwidth. For a normal distribution with standard deviation $\sigma_{\mathrm{G}}^{\mathrm{par}}$, the optimal bandwidth, $\lambda_{G}^{\mathrm{par}}$, is given by (Keen 2010) 


$$
\lambda_{G}^{\mathrm{par}} \approx 1.06 \sigma_{\mathrm{G}}^{\mathrm{par}} n_{\mathrm{pix}}^{-1 / 5}
$$

where $n_{\mathrm{pix}}$ is the number of pixels in our set with standard deviation $\sigma_{\mathrm{G}}^{\mathrm{par}}$. We then convolve the resulting histogram of the pixel population with a Gaussian function having a standard deviation $\sigma_{\text {conv }}=\lambda_{G}^{\mathrm{par}} / b_{w}^{\mathrm{par}}$. In the convolution, the Gaussian Kernel extends to $3 \sigma_{\text {conv }}$. When building the histogram of the pixel population, all pixels have the same weight.

\section{REFERENCES}

Abraham, R. G., Ellis, R. S., Fabian, A. C., Tanvir, N. R., \& Glazebrook, K. 1999, MNRAS, 303, 641

Aihara, H., Allende Prieto, C., An, D., et al. 2011, ApJS, 193, 29 Alam, S., Albareti, F. D., Allende Prieto, C., et al. 2015, ApJS, 219, 12

Benítez, N. 2000, ApJ, 536, 571

Bell, E. F., \& de Jong, R. S. 2001, ApJ, 550, 212

Bell, E. F., McIntosh, D. H., Katz, N., \& Weinberg, M. D. 2003, ApJS, 149, 289

Bershady, M. A., Verheijen, M. A. W., Swaters, R. A., et al. 2010, ApJ, 716, 198

Bertin, E. 2010, Astrophysics Source Code Library, ascl:1010.068

Bevington, P. R. 1969, Data reduction and error analysis for the physical sciences, New York: McGraw-Hill

Bhavsar, S. P. 1990, Errors, Bias and Uncertainties in Astronomy, 107

Blanton, M. R., \& Roweis, S. 2007, AJ, 133, 734

Block, D. L., \& Wainscoat, R. J. 1991, Nature, 353, 48

Block, D. L., Bertin, G., Stockton, A., et al. 1994, A\&A, 288, 365

Bothun, G. D. 1986, AJ, 91, 507

Bruzual, G., \& Charlot, S. 2003, MNRAS, 344, 1000

Bruzual, A. G. 2007, in IAU Symp. 241, Stellar Populations as Building Blocks of Galaxies, ed. A. Vazdekis \& R. F. Peletier (Cambridge: Cambridge Univ. Press), 241, 125

Bundy, K., Bershady, M. A., Law, D. R., et al. 2015, ApJ, 798, 7

Cappellari, M., \& Copin, Y. 2003, MNRAS, 342, 345

Chapman, N. L., Mundy, L. G., Lai, S.-P., \& Evans, N. J., II 2009, ApJ, 690, 49

Chabrier, G. 2003, PASP, 115, 763

Charlot, S., \& Fall, S. M. 2000, ApJ, 539, 718

Chen, Y.-M., Kauffmann, G., Tremonti, C. A., et al. 2012, MNRAS, 421, 314

Conroy, C., Gunn, J. E., \& White, M. 2009, ApJ, 699, 486

Conti, A., Connolly, A. J., Hopkins, A. M., et al. 2003, AJ, 126, 2330

Courteau, S., Cappellari, M., de Jong, R. S., et al. 2014, Reviews of Modern Physics, 86, 47

da Cunha, E., Charlot, S., \& Elbaz, D. 2008, MNRAS, 388, 1595

Daddi, E., Dickinson, M., Morrison, G., et al. 2007, ApJ, 670, 156 de Jong, R. S. 1996, A\&A, 313, 377

de Jong, R. S., \& Bell, E. F. 2007, Astrophysics and Space Science Proceedings, 3, 107

de Vaucouleurs, G., de Vaucouleurs, A., Corwin, H. G., Jr., et al 1991, Third Reference Catalogue of Bright Galaxies (RC3)

Driver, S. P., Popescu, C. C., Tuffs, R. J., et al. 2007, MNRAS, 379, 1022

Egusa, F., Mentuch Cooper, E., Koda, J., \& Baba, J. 2016, arXiv: 1610.06642

Elbaz, D., Daddi, E., Le Borgne, D., et al. 2007, A\&A, 468, 33

Eskridge, P. B., Frogel, J. A., Pogge, R. W., et al. 2002, ApJS, 143,73

Eskridge, P. B., Frogel, J. A., Taylor, V. A., et al. 2003, ApJ, 586, 923

Foyle, K., Rix, H.-W., \& Zibetti, S. 2010, MNRAS, 407, 163

Gallazzi, A., \& Bell, E. F. 2009, ApJS, 185, 253

Gonzalez, R. A., \& Graham, J. R. 1996, ApJ, 460, 651

Grosbøl, P., \& Dottori, H. 2008, A\&A, 490, 87

Grosbøl, P., Dottori, H., \& Gredel, R. 2006, A\&A, 453, L25

Gumus, K., \& Sen, A. 2013, Geodetski Vestnik, 57-3, 523

Into, T., \& Portinari, L. 2013, MNRAS, 430, 2715

James, P. A., \& Seigar, M. S. 1999, A\&A, 350, 791

Jarrett, T. H., Chester, T., Cutri, R., Schneider, S. E., \& Huchra, J. P. 2003, AJ, 125,525

Just, A., Fuchs, B., Jahreiß, H., et al. 2015, MNRAS, 451, 149

Kassin, S. A., Frogel, J. A., Pogge, R. W., Tiede, G. P., \& Sellgren, K. 2003, AJ, 126, 1276

Kassin, S. A., de Jong, R. S., \& Pogge, R. W. 2006, ApJS, 162, 80

Keen, K. J., Graphics for Statistics and Data Analysis with R, Chapman \& Hall/CRC, 2010

Kennicutt, R. C., Jr., Armus, L., Bendo, G., et al. 2003, PASP, 115,928

Kobulnicky, H. A., \& Kewley, L. J. 2004, ApJ, 617, 240

Kroupa, P. 2001, MNRAS, 322, 231
Lanyon-Foster, M. M., Conselice, C. J., \& Merrifield, M. R. 2007, MNRAS, 380,571

Leauthaud, A., George, M. R., Behroozi, P. S., et al. 2012, ApJ, 746,95

Lepage, R., \& Billard, L. 1992, Wiley Series in Probability and Mathematical Statistics, New York: Wiley, 1992

Leroy, A. K., Walter, F., Brinks, E., et al. 2008, AJ, 136, 2782

Loredo, T. J. 1992, Statistical Challenges in Modern Astronomy, 275

Loredo, T. J. 1995, Ph.D. Thesis, Univ. Chicago

Magris C., G., Mateu P., J., Mateu, C., et al. 2015, PASP, 127, 16

Maller, A. H., Berlind, A. A., Blanton, M. R., \& Hogg, D. W. 2009, ApJ, 691, 394

Maraston, C., Daddi, E., Renzini, A., et al. 2006, ApJ, 652, 85

Maraston, C., Pforr, J., Renzini, A., et al. 2010, MNRẢS, 407, 830

Martínez-García, E. E., González-Lópezlira, R. A., \& Bruzual-A, G. 2009, ApJ, 694, 512

Martínez-García, E. E., \& González-Lópezlira, R. A. 2013, ApJ, 765,105

Martinsson, T. P. K., Verheijen, M. A. W., Westfall, K. B., et al. 2013, A\&A, 557, A131

McGaugh, S. S., \& Schombert, J. M. 2014, AJ, 148, 77

McGaugh, S., Lelli, F., \& Schombert, J. 2016, Phys. Rev. Lett., 117,201101

Mei, S., Blakeslee, J. P., Côté, P., et al. 2007, ApJ, 655, 144

Meidt, S. E., Schinnerer, E., Knapen, J. H., et al. 2012, ApJ, 744 17

Meidt, S. E., Schinnerer, E., van de Ven, G., et al. 2014, ApJ, 788,144

Mentuch Cooper, E., Wilson, C. D., Foyle, K., et al. 2012, ApJ, 755,165

Mitchell, P. D., Lacey, C. G., Baugh, C. M., \& Cole, S. 2013, MNRAS, 435,87

Moustakas, J., Kennicutt, R. C., Jr., Tremonti, C. A., et al. 2010, ApJS, 190, 233-266

Noeske, K. G., Weiner, B. J., Faber, S. M., et al. 2007, ApJ, 660, L43

Padmanabhan, N., Schlegel, D. J., Finkbeiner, D. P., et al. 2008, ApJ, 674, 1217-1233

Patsis, P. A., Héraudeau, P., \& Grosb $\varnothing 1$, P. 2001, A\&A, 370, 875

Pilyugin, L. S., \& Thuan, T. X. 2005, ApJ, 631, 231

Portinari, L., Sommer-Larsen, J., \& Tantalo, R. 2004, MNRAS, 347, 691

Querejeta, M., Meidt, S. E., Schinnerer, E., et al. 2015, ApJS, 219,5

Reach, W. T., Megeath, S. T., Cohen, M., et al. 2005, PASP, 117, 978

Repetto, P., Martínez-García, E. E., Rosado, M., \& Gabbasov, R. 2013, ApJ, 765, 7

.2015, MNRAS, 451, 353

Rhoads, J. E. 1998, AJ, 115, 472

Rix, H.-W., \& Rieke, M. J. 1993, ApJ, 418, 123

Roediger, J. C., \& Courteau, S. 2015, MNRAS, 452, 3209

Rovilos, E., Georgantopoulos, I., Akylas, A., et al. 2014, MNRAS, 438, 494

Salpeter, E. E. 1955, ApJ, 121, 161

Salim, S., Rich, R. M., Charlot, S., et al. 2007, ApJS, 173, 267

Sánchez, S. F., Kennicutt, R. C., Gil de Paz, A., et al. 2012, A\&A, 538, A8

Savage, R. S., \& Oliver, S. 2007, ApJ, 661, 1339

Schlafly, E. F., \& Finkbeiner, D. P. 2011, ApJ, 737, 103

Schönrich, R., \& Bergemann, M. 2014, MNRAS, 443, 698

Sheth, K., Regan, M., Hinz, J. L., et al. 2010, PASP, 122, 1397

Skrutskie, M. F., Cutri, R. M., Stiening, R., et al. 2006, AJ, 131, 1163

Sorba, R., \& Sawicki, M. 2015, MNRAS, 452, 235

Sorce, J. G., Tully, R. B., Courtois, H. M., et al. 2014, MNRAS, 444,527

Taylor, E. N., Hopkins, A. M., Baldry, I. K., et al. 2011, MNRAS, 418, 1587

Tody, D. 1993, in ASP Conf. Ser. 52, Astronomical Data Analysis Software and Systems II, ed. R. J. Hanisch, R. J. V. Brissenden, \& J. Barnes (San Francisco, CA: ASP), 173 
Tikhonov, N. A., Galazutdinova, O. A., \& Tikhonov, E. N. 2009, Astronomy Letters, 35, 599

Welikala, N., Connolly, A. J., Hopkins, A. M., Scranton, R., \& Conti, A. 2008, ApJ, 677, 970

Wuyts, S., Förster Schreiber, N. M., Genzel, R., et al. 2012, ApJ, 753,114
Zibetti, S. 2009, arXiv:0911.4956

Zibetti, S., Charlot, S., \& Kix, H.-W. 2009, MNRAS, 400, 1181, ZCR

TABLE 1

GALAXY PARAMETERS

\begin{tabular}{|c|c|c|c|c|c|c|c|}
\hline Name & RC3 type & T-type & Dist (Mpc) & $\Upsilon_{*}^{H}$ & $M_{*}^{\mathrm{BSP}}\left(M_{\odot}\right)$ & $M_{*}^{\mathrm{ZCR}^{\prime}}\left(M_{\odot}\right)$ & $M_{*}^{\text {unresolved }}\left(M_{\odot}\right)$ \\
\hline M 51 & $\mathrm{SA}(\mathrm{s}) \mathrm{bc}$ pec & 4.0 & $9.9^{\mathrm{a}} \pm 0.7$ & $0.42\left(K_{s}\right)$ & $5.56 \times 10^{10}$ & $6.43 \times 10^{10}$ & $(9.02 \pm 3.00) \times 10^{10}$ \\
\hline M 51b & I0 pec & 90.0 & $9.9^{\mathrm{a}} \pm 0.7$ & $0.97\left(K_{s}\right)$ & $2.96 \times 10^{10 b}$ & $4.66 \times 10^{10 b}$ & $(3.26 \pm 0.22) \times 10^{10 b}$ \\
\hline NGC 157 & $\mathrm{SAB}(\mathrm{rs}) \mathrm{bc}$ & 4.0 & $22.6 \pm 1.6$ & 0.58 & $4.52 \times 10^{10}$ & $4.92 \times 10^{10}$ & $(3.96 \pm 0.85) \times 10^{10}$ \\
\hline NGC 428 & $\mathrm{SAB}(\mathrm{s}) \mathrm{m}$ & 9.0 & $15.9 \pm 1.1$ & 0.40 & $3.71 \times 10^{9}$ & $4.28 \times 10^{9}$ & $(3.80 \pm 0.61) \times 10^{9}$ \\
\hline NGC 488 & $\mathrm{SA}(\mathrm{r}) \mathrm{b}$ & 3.0 & $30.4 \pm 2.1$ & 1.04 & $2.61 \times 10^{11}$ & $2.60 \times 10^{11}$ & $(3.19 \pm 0.87) \times 10^{11}$ \\
\hline NGC 779 & $\mathrm{SAB}(\mathrm{r}) \mathrm{b}$ & 3.0 & $18.5 \pm 1.3$ & 0.72 & $2.69 \times 10^{10}$ & $3.13 \times 10^{10}$ & $(2.38 \pm 0.65) \times 10^{10}$ \\
\hline NGC 864 & $\mathrm{SAB}(\mathrm{rs}) \mathrm{c}$ & 5.0 & $20.9 \pm 1.5$ & 0.53 & $1.55 \times 10^{10}$ & $1.83 \times 10^{10}$ & $(1.85 \pm 0.34) \times 10^{10}$ \\
\hline NGC 1042 & $\mathrm{SAB}(\mathrm{rs}) \mathrm{cd}$ & 6.0 & $18.1 \pm 1.3$ & 0.56 & $1.20 \times 10^{10}$ & $1.43 \times 10^{10}$ & $(1.32 \pm 0.26) \times 10^{10}$ \\
\hline NGC 1073 & $\mathrm{SB}(\mathrm{rs}) \mathrm{c}$ & 5.0 & $16.1 \pm 1.1$ & 0.51 & $5.83 \times 10^{9}$ & $6.93 \times 10^{9}$ & $(6.90 \pm 1.23) \times 10^{9}$ \\
\hline NGC 1084 & $\mathrm{SA}(\mathrm{s}) \mathrm{c}$ & 5.0 & $18.6 \pm 1.3$ & 0.66 & $2.70 \times 10^{10}$ & $2.48 \times 10^{10}$ & $(2.17 \pm 0.40) \times 10^{10}$ \\
\hline NGC 1087 & $\mathrm{SAB}(\mathrm{rs}) \mathrm{c}$ & 5.0 & $20.1 \pm 1.4$ & 0.54 & $1.52 \times 10^{10}$ & $1.46 \times 10^{10}$ & $(1.30 \pm 0.25) \times 10^{10}$ \\
\hline NGC 1309 & $\mathrm{SA}(\mathrm{s}) \mathrm{bc}:$ & 4.0 & $28.3 \pm 2.0$ & 0.34 & $1.17 \times 10^{10}$ & $1.42 \times 10^{10}$ & $(1.13 \pm 0.18) \times 10^{10}$ \\
\hline NGC 2775 & $\mathrm{SA}(\mathrm{r}) \mathrm{ab}$ & 2.0 & $21.4 \pm 1.5$ & 1.12 & $1.34 \times 10^{11}$ & $1.26 \times 10^{11}$ & $(1.33 \pm 0.24) \times 10^{11}$ \\
\hline NGC 2964 & $\mathrm{SAB}(\mathrm{r}) \mathrm{bc}:$ & 4.0 & $23.2 \pm 1.6$ & 0.69 & $2.90 \times 10^{10}$ & $2.98 \times 10^{10}$ & $(2.59 \pm 0.60) \times 10^{10}$ \\
\hline NGC 3166 & $\mathrm{SAB}(\mathrm{rs}) 0 / \mathrm{a}$ & 0.0 & $22.0 \pm 1.5$ & 0.99 & $9.69 \times 10^{10}$ & $1.04 \times 10^{11}$ & $(5.87 \pm 2.69) \times 10^{10}$ \\
\hline NGC 3169 & $\mathrm{SA}(\mathrm{s}) \mathrm{a}$ pec & 1.0 & $19.9 \pm 1.4$ & 0.95 & $6.93 \times 10^{10}$ & $1.16 \times 10^{11}$ & $(5.07 \pm 1.76) \times 10^{10}$ \\
\hline NGC 3227 & $\mathrm{SAB}(\mathrm{s}) \mathrm{a}$ pec & 1.0 & $20.3 \pm 1.4$ & 1.03 & $4.74 \times 10^{10}$ & $5.01 \times 10^{10}$ & $(3.27 \pm 0.71) \times 10^{10}$ \\
\hline NGC 3319 & $\mathrm{SB}(\mathrm{rs}) \mathrm{cd}$ & 6.0 & $3.3 \pm 0.9$ & 0.48 & $1.21 \times 10^{8}$ & $1.37 \times 10^{8}$ & $(9.68 \pm 1.99) \times 10^{7}$ \\
\hline NGC 3338 & $\mathrm{SA}(\mathrm{s}) \mathrm{c}$ & 5.0 & $23.2 \pm 1.6$ & 0.47 & $2.02 \times 10^{10}$ & $2.88 \times 10^{10}$ & $(1.87 \pm 0.45) \times 10^{10}$ \\
\hline NGC 3423 & $\mathrm{SA}(\mathrm{s}) \mathrm{cd}$ & 6.0 & $14.1 \pm 1.0$ & 0.39 & $3.99 \times 10^{9}$ & $4.60 \times 10^{9}$ & $(4.42 \pm 0.53) \times 10^{9}$ \\
\hline NGC 3504 & (R)SAB(s)ab & 2.0 & $27.8 \pm 1.9$ & 0.69 & $4.56 \times 10^{10}$ & $4.71 \times 10^{10}$ & $(5.65 \pm 1.38) \times 10^{10}$ \\
\hline NGC 3507 & $\mathrm{SB}(\mathrm{s}) \mathrm{b}$ & 3.0 & $15.0 \pm 1.1$ & 0.56 & $8.92 \times 10^{9}$ & $1.20 \times 10^{10}$ & $(8.79 \pm 2.13) \times 10^{9}$ \\
\hline NGC 3583 & $\mathrm{SB}(\mathrm{s}) \mathrm{b}$ & 3.0 & $35.7 \pm 2.5$ & 0.67 & $6.25 \times 10^{10}$ & $7.80 \times 10^{10}$ & $(3.77 \pm 3.14) \times 10^{10}$ \\
\hline NGC 3593 & $\mathrm{SA}(\mathrm{s}) 0 / \mathrm{a}$ & 0.0 & $5.6 \pm 0.4$ & 1.04 & $5.11 \times 10^{9}$ & $6.65 \times 10^{9}$ & $(4.69 \pm 0.95) \times 10^{9}$ \\
\hline NGC 3596 & $\mathrm{SAB}(\mathrm{rs}) \mathrm{c}$ & 5.0 & $22.5 \pm 1.6$ & 0.54 & $1.38 \times 10^{10}$ & $1.51 \times 10^{10}$ & $(1.62 \pm 0.29) \times 10^{10}$ \\
\hline NGC 3646 & RING & 4.0 & $65.2 \pm 4.6$ & 0.52 & $1.43 \times 10^{11}$ & $2.05 \times 10^{11}$ & $(1.36 \pm 0.32) \times 10^{11}$ \\
\hline NGC 3675 & $\mathrm{SA}(\mathrm{s}) \mathrm{b}$ & 3.0 & $14.3 \pm 1.0$ & 1.21 & $7.50 \times 10^{10}$ & $7.00 \times 10^{10}$ & $(5.34 \pm 1.20) \times 10^{10}$ \\
\hline NGC 3681 & $\mathrm{SAB}(\mathrm{r}) \mathrm{bc}$ & 4.0 & $24.9 \pm 1.7$ & 0.90 & $2.40 \times 10^{10}$ & $2.23 \times 10^{10}$ & $(2.02 \pm 0.42) \times 10^{10}$ \\
\hline NGC 3684 & $\mathrm{SA}(\mathrm{rs}) \mathrm{bc}$ & 4.0 & $22.8 \pm 1.6$ & 0.81 & $1.16 \times 10^{10}$ & $1.11 \times 10^{10}$ & $(8.70 \pm 2.11) \times 10^{9}$ \\
\hline NGC 3686 & $\mathrm{SB}(\mathrm{s}) \mathrm{bc}$ & 4.0 & $22.6 \pm 1.6$ & 0.63 & $2.42 \times 10^{10}$ & $2.40 \times 10^{10}$ & $(2.36 \pm 0.47) \times 10^{10}$ \\
\hline NGC 3705 & $\mathrm{SAB}(\mathrm{r}) \mathrm{ab}$ & 2.0 & $13.2 \pm 0.9$ & 0.55 & $1.08 \times 10^{10}$ & $1.74 \times 10^{10}$ & $(1.04 \pm 0.33) \times 10^{10}$ \\
\hline NGC 3810 & $\mathrm{SA}(\mathrm{rs}) \mathrm{c}$ & 5.0 & $10.7 \pm 0.8$ & 0.49 & $5.27 \times 10^{9}$ & $7.11 \times 10^{9}$ & $(5.54 \pm 1.23) \times 10^{9}$ \\
\hline NGC 3877 & $\mathrm{SA}(\mathrm{s}) \mathrm{c}:$ & 5.0 & $17.8 \pm 1.3$ & 0.84 & $3.77 \times 10^{10}$ & $4.25 \times 10^{10}$ & $(2.72 \pm 1.34) \times 10^{10}$ \\
\hline NGC 3893 & $\mathrm{SAB}(\mathrm{rs}) \mathrm{c}:$ & 5.0 & $19.4 \pm 1.4$ & 0.53 & $2.36 \times 10^{10}$ & $2.41 \times 10^{10}$ & $(2.33 \pm 0.42) \times 10^{10}$ \\
\hline NGC 3938 & $\mathrm{SA}(\mathrm{s}) \mathrm{c}$ & 5.0 & $15.5 \pm 1.1$ & 0.50 & $1.56 \times 10^{10}$ & $1.79 \times 10^{10}$ & $(2.13 \pm 0.57) \times 10^{10}$ \\
\hline NGC 3949 & SA(s)bc: & 4.0 & $15.8 \pm 1.1$ & 0.69 & $1.17 \times 10^{10}$ & $8.20 \times 10^{9}$ & $(6.06 \pm 1.02) \times 10^{9}$ \\
\hline NGC 4030 & $\mathrm{SA}(\mathrm{s}) \mathrm{bc}$ & 4.0 & $26.4 \pm 1.8$ & 0.61 & $7.93 \times 10^{10}$ & $9.38 \times 10^{10}$ & $(5.54 \pm 3.21) \times 10^{10}$ \\
\hline NGC 4051 & $\mathrm{SAB}(\mathrm{rs}) \mathrm{bc}$ & 4.0 & $2.9 \pm 0.9$ & 0.68 & $7.80 \times 10^{8}$ & $8.55 \times 10^{8}$ & $(6.34 \pm 1.52) \times 10^{8}$ \\
\hline NGC 4062 & $\mathrm{SA}(\mathrm{s}) \mathrm{c}$ & 5.0 & $10.4 \pm 0.7$ & 0.67 & $7.07 \times 10^{9}$ & $8.34 \times 10^{9}$ & $(6.03 \pm 1.59) \times 10^{9}$ \\
\hline NGC 4100 & $\left(\mathrm{R}^{\prime}\right) \mathrm{SA}(\mathrm{rs}) \mathrm{bc}$ & 4.0 & $21.5 \pm 1.5$ & 0.72 & $3.27 \times 10^{10}$ & $4.02 \times 10^{10}$ & $(2.74 \pm 0.73) \times 10^{10}$ \\
\hline NGC 4123 & $\mathrm{SB}(\mathrm{r}) \mathrm{c}$ & 5.0 & $27.3 \pm 1.9$ & 0.55 & $2.09 \times 10^{10}$ & $2.55 \times 10^{10}$ & $(1.78 \pm 0.43) \times 10^{10}$ \\
\hline NGC 4136 & $\mathrm{SAB}(\mathrm{r}) \mathrm{c}$ & 5.0 & $6.7 \pm 0.5$ & 0.42 & $6.51 \times 10^{8}$ & $7.42 \times 10^{8}$ & $(4.42 \pm 2.12) \times 10^{8}$ \\
\hline NGC 4145 & $\mathrm{SAB}(\mathrm{rs}) \mathrm{d}$ & 7.0 & $20.3 \pm 1.4$ & 0.53 & $1.38 \times 10^{10}$ & $1.51 \times 10^{10}$ & $(1.01 \pm 0.31) \times 10^{10}$ \\
\hline NGC 4151 & $\left(\mathrm{R}^{\prime}\right) \mathrm{SAB}(\mathrm{rs}) \mathrm{ab}$ : & 2.0 & $20.0 \pm 1.4$ & 0.98 & $4.29 \times 10^{10}$ & $4.21 \times 10^{10}$ & $(3.30 \pm 0.58) \times 10^{10}$ \\
\hline NGC 4212 & SAc: & 4.5 & $16.3^{\mathrm{c}} \pm 3.8$ & 0.68 & $1.64 \times 10^{10}$ & $1.65 \times 10^{10}$ & $(1.76 \pm 0.33) \times 10^{10}$ \\
\hline NGC 4254 & $\mathrm{SA}(\mathrm{s}) \mathrm{c}$ & 5.0 & $16.5^{\mathrm{d}} \pm 1.1$ & 0.46 & $3.37 \times 10^{10}$ & $4.29 \times 10^{10}$ & $(3.70 \pm 0.71) \times 10^{10}$ \\
\hline NGC 4293 & $(\mathrm{R}) \mathrm{SB}(\mathrm{s}) 0 / \mathrm{a}$ & 0.0 & $14.1 \pm 1.0$ & 0.85 & $2.93 \times 10^{10}$ & $3.39 \times 10^{10}$ & $(3.61 \pm 0.89) \times 10^{10}$ \\
\hline NGC 4303 & $\mathrm{SAB}(\mathrm{rs}) \mathrm{bc}$ & 4.0 & $13.6 \pm 1.0$ & 0.56 & $2.80 \times 10^{10}$ & $3.06 \times 10^{10}$ & $(2.32 \pm 0.50) \times 10^{10}$ \\
\hline NGC 4314 & $\mathrm{SB}(\mathrm{rs}) \mathrm{a}$ & 1.0 & $17.8 \pm 1.3$ & 1.03 & $6.03 \times 10^{10}$ & $5.55 \times 10^{10}$ & $(4.34 \pm 1.01) \times 10^{10}$ \\
\hline NGC 4388 & $\mathrm{SA}(\mathrm{s}) \mathrm{b}: \mathrm{sp}$ & 3.0 & $41.4 \pm 2.9$ & 0.64 & $1.08 \times 10^{11}$ & $1.28 \times 10^{11}$ & $(1.55 \pm 0.43) \times 10^{11}$ \\
\hline NGC 4394 & (R) SB $(\mathrm{r}) \mathrm{b}$ & 3.0 & $14.1 \pm 1.0$ & 0.77 & $1.76 \times 10^{10}$ & $1.94 \times 10^{10}$ & $(1.74 \pm 0.35) \times 10^{10}$ \\
\hline NGC 4414 & $\mathrm{SA}(\mathrm{rs}) \mathrm{c} ?$ & 5.0 & $9.0 \pm 0.6$ & 0.74 & $1.68 \times 10^{10}$ & $1.99 \times 10^{10}$ & $(1.67 \pm 0.39) \times 10^{10}$ \\
\hline NGC 4448 & $\mathrm{SB}(\mathrm{r}) \mathrm{ab}$ & 2.0 & $7.0 \pm 0.5$ & 0.89 & $5.61 \times 10^{9}$ & $5.98 \times 10^{9}$ & $(4.66 \pm 1.16) \times 10^{9}$ \\
\hline NGC 4450 & $\mathrm{SA}(\mathrm{s}) \mathrm{ab}$ & 2.0 & $14.1 \pm 1.0$ & 1.07 & $5.09 \times 10^{10}$ & $4.85 \times 10^{10}$ & $(4.66 \pm 0.71) \times 10^{10}$ \\
\hline NGC 4457 & (R)SAB(s) $0 / a$ & 0.0 & $13.6 \pm 1.0$ & 1.03 & $2.18 \times 10^{10}$ & $2.18 \times 10^{10}$ & $(1.91 \pm 0.23) \times 10^{10}$ \\
\hline NGC 4490 & $\mathrm{SB}(\mathrm{s}) \mathrm{d}$ pec & 7.0 & $9.2 \pm 0.7$ & 0.38 & $6.08 \times 10^{9}$ & $5.91 \times 10^{9}$ & $(4.69 \pm 0.75) \times 10^{9}$ \\
\hline NGC 4496A & $\mathrm{SB}(\mathrm{rs}) \mathrm{m}$ & 9.0 & $13.6 \pm 1.4$ & 0.43 & $2.31 \times 10^{9}$ & $2.59 \times 10^{9}$ & $(2.62 \pm 0.42) \times 10^{9}$ \\
\hline NGC 4527 & $\mathrm{SAB}(\mathrm{s}) \mathrm{bc}$ & 4.0 & $13.5 \pm 0.9$ & 0.81 & $3.39 \times 10^{10}$ & $5.93 \times 10^{10}$ & $(3.85 \pm 0.95) \times 10^{10}$ \\
\hline NGC 4548 & $\mathrm{SB}(\mathrm{rs}) \mathrm{b}$ & 3.0 & $3.7 \pm 0.3$ & 0.95 & $3.03 \times 10^{9}$ & $3.10 \times 10^{9}$ & $(2.73 \pm 0.49) \times 10^{9}$ \\
\hline NGC 4568 & $\mathrm{SA}(\mathrm{rs}) \mathrm{bc}$ & 4.0 & $13.9 \pm 1.0$ & 0.75 & $2.15 \times 10^{10}$ & $3.02 \times 10^{10}$ & $(2.19 \pm 0.59) \times 10^{10}$ \\
\hline NGC 4571 & $\mathrm{SA}(\mathrm{r}) \mathrm{d}$ & 6.5 & $2.6 \pm 0.2$ & 0.82 & $3.91 \times 10^{8}$ & $3.99 \times 10^{8}$ & $(2.72 \pm 0.66) \times 10^{8}$ \\
\hline NGC 4579 & $\mathrm{SAB}(\mathrm{rs}) \mathrm{b}$ & 3.0 & $13.9 \pm 1.0$ & 1.00 & $7.76 \times 10^{10}$ & $7.44 \times 10^{10}$ & $(6.19 \pm 1.19) \times 10^{10}$ \\
\hline
\end{tabular}


TABLE 1 - Continued

\begin{tabular}{|c|c|c|c|c|c|c|c|}
\hline Name & RC3 type & T-type & Dist (Mpc) & $\Upsilon_{*}^{H}$ & $M_{*}^{\mathrm{BSP}}\left(M_{\odot}\right)$ & $M_{*}^{\mathrm{ZCR}}\left(M_{\odot}\right)$ & $M_{*}^{\text {unresolved }}\left(M_{\odot}\right)$ \\
\hline NGC 4580 & $\mathrm{SAB}(\mathrm{rs})$ a pec & 1.0 & $13.6 \pm 1.0$ & 0.93 & $9.26 \times 10^{9}$ & $9.05 \times 10^{9}$ & $(5.60 \pm 2.19) \times 10^{9}$ \\
\hline NGC 4618 & $\mathrm{SB}(\mathrm{rs}) \mathrm{m}$ & 9.0 & $8.8 \pm 0.6$ & 0.48 & $2.76 \times 10^{9}$ & $2.54 \times 10^{9}$ & $(1.64 \pm 0.59) \times 10^{9}$ \\
\hline NGC 4643 & $\mathrm{SB}(\mathrm{rs}) 0 / \mathrm{a}$ & 0.0 & $27.3 \pm 1.9$ & 1.22 & $1.71 \times 10^{11}$ & $1.74 \times 10^{11}$ & $(1.64 \pm 0.12) \times 10^{11}$ \\
\hline NGC 4647 & $\mathrm{SAB}(\mathrm{rs}) \mathrm{c}$ & 5.0 & $13.9 \pm 1.0$ & 0.97 & $2.11 \times 10^{10}$ & $1.92 \times 10^{10}$ & $(1.66 \pm 0.32) \times 10^{10}$ \\
\hline NGC 4651 & $\mathrm{SA}(\mathrm{rs}) \mathrm{c}$ & 5.0 & $14.0 \pm 1.0$ & 0.59 & $1.37 \times 10^{10}$ & $1.63 \times 10^{10}$ & $(1.05 \pm 0.34) \times 10^{10}$ \\
\hline NGC 4654 & $\mathrm{SAB}(\mathrm{rs}) \mathrm{cd}$ & 6.0 & $13.9 \pm 1.0$ & 0.55 & $1.34 \times 10^{10}$ & $1.60 \times 10^{10}$ & $(1.09 \pm 0.24) \times 10^{10}$ \\
\hline NGC 4665 & $\mathrm{SB}(\mathrm{s}) 0 / \mathrm{a}$ & 0.0 & $13.5 \pm 0.9$ & 0.88 & $4.76 \times 10^{10}$ & $5.39 \times 10^{10}$ & $(4.80 \pm 1.14) \times 10^{10}$ \\
\hline NGC 4666 & SABc: & 5.0 & $27.5 \pm 1.9$ & 0.94 & $1.45 \times 10^{11}$ & $1.87 \times 10^{11}$ & $(1.91 \pm 0.45) \times 10^{11}$ \\
\hline NGC 4689 & $\mathrm{SA}(\mathrm{rs}) \mathrm{bc}$ & 4.0 & $14.0 \pm 1.0$ & 0.74 & $1.47 \times 10^{10}$ & $1.45 \times 10^{10}$ & $(1.07 \pm 0.28) \times 10^{10}$ \\
\hline NGC 4691 & (R)SB(s)0/a pec & 0.0 & $17.0 \pm 1.2$ & 0.83 & $1.95 \times 10^{10}$ & $1.57 \times 10^{10}$ & $(1.25 \pm 0.27) \times 10^{10}$ \\
\hline NGC 4698 & $\mathrm{SA}(\mathrm{s}) \mathrm{ab}$ & 2.0 & $13.7 \pm 1.0$ & 1.13 & $3.46 \times 10^{10}$ & $3.40 \times 10^{10}$ & $(3.12 \pm 0.45) \times 10^{10}$ \\
\hline NGC 4699 & $\mathrm{SAB}(\mathrm{rs}) \mathrm{b}$ & 3.0 & $22.9 \pm 1.6$ & 1.16 & $2.02 \times 10^{11}$ & $1.58 \times 10^{11}$ & $(1.28 \pm 0.27) \times 10^{11}$ \\
\hline NGC 4772 & $\mathrm{SA}(\mathrm{s}) \mathrm{a}$ & 1.0 & $13.3 \pm 0.9$ & 1.04 & $1.66 \times 10^{10}$ & $1.61 \times 10^{10}$ & $(1.32 \pm 0.24) \times 10^{10}$ \\
\hline NGC 4900 & $\mathrm{SB}(\mathrm{rs}) \mathrm{c}$ & 5.0 & $9.1 \pm 0.6$ & 0.55 & $2.30 \times 10^{9}$ & $2.15 \times 10^{9}$ & $(2.38 \pm 0.53) \times 10^{9}$ \\
\hline NGC 5005 & $\mathrm{SAB}(\mathrm{rs}) \mathrm{bc}$ & 4.0 & $19.3 \pm 1.4$ & 0.82 & $1.25 \times 10^{11}$ & $1.49 \times 10^{11}$ & $(1.83 \pm 0.51) \times 10^{11}$ \\
\hline NGC 5334 & $\mathrm{SB}(\mathrm{rs}) \mathrm{c}$ & 5.0 & $24.2 \pm 1.7$ & 0.59 & $1.11 \times 10^{10}$ & $1.24 \times 10^{10}$ & $(8.32 \pm 2.23) \times 10^{9}$ \\
\hline NGC 5371 & $\mathrm{SAB}(\mathrm{rs}) \mathrm{bc}$ & 4.0 & $42.8 \pm 3.0$ & 0.90 & $1.60 \times 10^{11}$ & $1.74 \times 10^{11}$ & $(1.29 \pm 0.21) \times 10^{11}$ \\
\hline NGC 5448 & (R)SAB $(r) a$ & 1.0 & $35.2 \pm 2.5$ & 0.81 & $5.86 \times 10^{10}$ & $7.11 \times 10^{10}$ & $(5.67 \pm 1.23) \times 10^{10}$ \\
\hline NGC 5676 & $\mathrm{SA}(\mathrm{rs}) \mathrm{bc}$ & 4.0 & $36.5 \pm 2.6$ & 0.64 & $9.23 \times 10^{10}$ & $1.16 \times 10^{11}$ & $(1.20 \pm 0.23) \times 10^{11}$ \\
\hline NGC 5701 & (R) SB $(r s) 0 / a$ & 0.0 & $26.7 \pm 1.9$ & 0.92 & $6.33 \times 10^{10}$ & $6.77 \times 10^{10}$ & $(6.32 \pm 1.08) \times 10^{10}$ \\
\hline NGC 5713 & $\mathrm{SAB}(\mathrm{rs}) \mathrm{bc}$ pec & 4.0 & $31.3 \pm 2.2$ & 0.63 & $4.93 \times 10^{10}$ & $4.95 \times 10^{10}$ & $(3.96 \pm 0.86) \times 10^{10}$ \\
\hline NGC 5850 & $\mathrm{SB}(\mathrm{r}) \mathrm{b}$ & 3.0 & $41.6 \pm 2.9$ & 0.74 & $1.41 \times 10^{11}$ & $1.78 \times 10^{11}$ & $(1.38 \pm 0.32) \times 10^{11}$ \\
\hline NGC 5921 & $\mathrm{SB}(\mathrm{r}) \mathrm{bc}$ & 4.0 & $26.2 \pm 1.8$ & 0.53 & $3.68 \times 10^{10}$ & $4.66 \times 10^{10}$ & $(4.40 \pm 0.83) \times 10^{10}$ \\
\hline NGC 5962 & $\mathrm{SA}(\mathrm{r}) \mathrm{c}$ & 5.0 & $34.2 \pm 2.4$ & 0.50 & $4.37 \times 10^{10}$ & $5.83 \times 10^{10}$ & $(6.85 \pm 1.67) \times 10^{10}$ \\
\hline NGC 6384 & $\mathrm{SAB}(\mathrm{r}) \mathrm{bc}$ & 4.0 & $29.2 \pm 2.0$ & 0.68 & $8.62 \times 10^{10}$ & $1.13 \times 10^{11}$ & $(7.26 \pm 1.86) \times 10^{10}$ \\
\hline NGC 7217 & (R)SA(r)ab & 2.0 & $16.5 \pm 1.2$ & 1.10 & $9.60 \times 10^{10}$ & $9.20 \times 10^{10}$ & $(1.02 \pm 0.22) \times 10^{11}$ \\
\hline NGC 7479 & $\mathrm{SB}(\mathrm{s}) \mathrm{c}$ & 5.0 & $33.7 \pm 2.4$ & 0.58 & $8.31 \times 10^{10}$ & $1.06 \times 10^{11}$ & $(7.82 \pm 1.92) \times 10^{10}$ \\
\hline NGC 7606 & $\mathrm{SA}(\mathrm{s}) \mathrm{b}$ & 3.0 & $31.3 \pm 2.2$ & 0.66 & $9.48 \times 10^{10}$ & $1.17 \times 10^{11}$ & $(1.52 \pm 0.35) \times 10^{11}$ \\
\hline NGC 7741 & $\mathrm{SB}(\mathrm{s}) \mathrm{cd}$ & 6.0 & $12.5 \pm 0.9$ & 0.50 & $3.47 \times 10^{9}$ & $3.66 \times 10^{9}$ & $(2.82 \pm 0.56) \times 10^{9}$ \\
\hline NGC 7814 & $\mathrm{SA}(\mathrm{s}) \mathrm{ab}: \mathrm{sp}$ & 2.0 & $15.7 \pm 1.1$ & 1.34 & $6.24 \times 10^{10 \mathrm{~b}}$ & $9.08 \times 10^{10 b}$ & $(6.21 \pm 0.52) \times 10^{10 b}$ \\
\hline
\end{tabular}

Note. - Col. 1: galaxy name. Col. 2: RC3 type de Vaucouleurs et al. 1991). Col. 3: T Hubble type (de Vaucouleurs et al. 1991). Col. 4: distance to object in Mpc, from NED (Virgo + GA + Shapley), unless otherwise indicated. Col. 5: median $\Upsilon_{*}^{H}$ after BSP iteration number 1 . For M 51 and M 51b the median $\Upsilon_{*}^{K_{s}^{*}}$ is tabulated instead of $\Upsilon_{*}^{H}$. Col. 6: total resolved stellar mass obtained from the BSP algorithm, $M_{*}^{\text {BSP }}$, in solar units. Col. 7: total resolved stellar mass obtained from $\mathrm{ZCR}^{\prime}, M_{*}^{\mathrm{ZCR}}$, in solar units. Col. 8: unresolved stellar mass, $M_{*}^{\text {unresolved }}$, in solar units. All the masses given in this table have been calculated using the SSAG-BC03 SPS library. The uncertainties in $M_{*}^{\text {unresolved }}$ correspond to the propagation of the systematic error due to the zero point calibration, which affects the values of $M_{*}^{\mathrm{BSP}}$ and $M_{*}^{Z C R^{\prime}}$ by only $\sim 3 \%$. The systematic uncertainty in the distance to the objects is not quoted in this table (see section 6).

a Tikhonov et al. 2009)

b Lower limit.

c Sorce et al. (2014)

d Mei et al. (2007) 